\title{
Complex dynamic behavior of a rumor propagation model with spatial-temporal diffusion terms
}

\author{
Linhe Zhu ${ }^{\mathrm{a}}$, Hongyong Zhao $\dagger^{\mathrm{a}}$, Haiyan Wang ${ }^{\mathrm{b}}$ \\ ${ }^{a}$ Department of Mathematics, Nanjing University of Aeronautics and Astronautics, Nanjing 210016, China. \\ ${ }^{b}$ School of Mathematical and Natural Sciences, Arizona State University, Phoenix AZ 85069-7100, USA.
}

\begin{abstract}
Rumor propagation as a typical form of social communication in online social networks has had a significant negative impact on a harmonious and stable society. With the rapid development of mobile communication equipments, traditional rumor propagation models, which depend on ordinary differential equations (ODE), may not be suitable for describing rumor propagation in an online social network. In this paper, based on reaction-diffusion equations, we propose a novel epidemic-like model with both discrete and nonlocal delays for investigating the spatial-temporal dynamics of rumor propagation. By analyzing the corresponding characteristic equations of this model, the local stability conditions of a boundary equilibrium point and a positive equilibrium point are established. By applying the linear approximation method of nonlinear systems, sufficient conditions are derived for the existence of Hopf bifurcation at the above two kinds of equilibrium points. Moreover, a sensitivity analysis method based on the density of spreading users is proposed, and then in theoretical and experimental aspect we identify some sensitive parameters in the process of rumor propagation. Finally, numerical simulations are performed to illustrate the theoretical results.
\end{abstract}

Keywords: Delay, Online social networks, Reaction-diffusion equations, Rumor propagation, Stability.

\section{Introduction}

Online social networks, such as Facebook, Twitter, Digg, the blogosphere and so on, allow hundreds of millions of Internet users worldwide to produce and consume content $[1,7,12,13$, $16,25,38]$. Such networks provide access to a vast information space on an unprecedented scale. With the popularization of online social networks, more and more Internet users, for their own purposes which may include diverting attention, manufacturing momentum, causing panic, harming national honor and interests and so on, have begun to spread harmful information through online social networks. For example, in the past decade, rumor propagation not only in small-world networks $[4,39,40]$ but also in scale-free networks [21, 22] has attracted much attention from physical and sociological research communities $[6,11,17]$. Rumor is an important

Email addresses: zlhnuaa@126.com (Linhe Zhu), hyzhao1967@126.com (Hongyong Zhao †), Haiyan. Wang@asu. edu (Haiyan Wang)

Preprint submitted to Information Sciences

February 11, 2016

(C) 2016. This manuscript version is made available under the Elsevier user license http://www.elsevier.com/open-access/userlicense/1.0/ 
form of social communication, and its propagation plays a significant negative role in a harmonious and stable society. Hayakawa [8] defines rumor as fact-like but unconfirmed information about some event, which can quickly spread through chains of communication on a large scale. Shibutani [27] regards rumor as collective problem-solving, in which people caught in ambiguous situations try to construe a meaningful interpretation by pooling their intellectual resources. Recently, Zhao [33] considers that rumor is a kind of visionary, mostly based not on objectivity but on subjective willingness and fabricated message. Usually, rumor involves influential events such as political and economic issues or public interesting news. Compared with the word-ofmouth way of rumor propagation of past times, rumor propagation in online social networks has become more flexible, faster, and more destructive. Convenient and efficient means to spread rumor may cause panic as well as economic loss resulting from the accompanying unexpected events [28]. These seriously threaten the stability of the society.

To reduce or eliminate the danger of the rumor propagation in online social networks, it is necessary to adequately understand the dynamic characteristics of rumor propagation. Mathematical modeling, an efficient tool, has been used to reveal many phenomena, including the dissemination of gossip, rumors or information. To the best of our knowledge, research engaging rumor propagation models started during the 1960s. In 1964, Daley and Kendall [2, 3], divided agents into three classes: ignorants, spreaders, and stiflers, and then they proposed the first classical rumor propagation model, which was called DK model by the later scholars. In fact, the DK model together with its variants, such as the Maki-Thompson (MK) model [20], are conceptually similar to the classical SIR (susceptible-infected-recovered) epidemic models. Given the similarities of rumor propagation and epidemic diffusion, many researchers since then have used the epidemiological model to describe information or rumor propagation on the complex networks [10, 18, 19, 34, 35, 44, 45, 46, 47, 49]. Zanette, based on static [40] and dynamic [39] small-world networks, respectively provided simulations of the deterministic MK model. Later, by means of Monte Carlo simulations and numerical solutions of a set of mean-field equations, Moreno et al. [21, 22] considered the stochastic version of the MK model on scale-free networks. Recently, references $[10,46]$ based on utility theory, both proposed a simple model to analyze the interplay between rumor propagation and authorities' actions in emergency situation. Zhao et al. [44, 45] provided a detailed description of rumor propagation by establishing a SIR epidemic-like model and a so-called SIHR model. Specifically, they considered the forgetting mechanism in this work. Further, combined with the network structure, Zhao et al. [47] discussed a rumor propagation model using mean-field equations with a variable forgetting rate. Their results showed that the final size of rumor propagation is much larger under a variable forgetting rate compared to that under a constant forgetting rate.

The existing models of rumor propagation in online social networks have concentrated only on the temporal dimension. Recently, a diffusive logistic (DL) model with a spatial-temporal diffusion term was proposed in [31] to study the information propagation process in online social networks. The authors described the spatial distance by using a new concept, friendship hops, and abstractly divided the information diffusion process in online social networks into two separate processes: a growth process and a social process. Wang et al. in [32] based on a partial differential equation (PDE), further proposed a linear diffusive model to understand the information diffusion process over both temporal and spatial dimensions. Combined with the empirical observations in the Digg data set, they proved the performance of the proposed linear diffusive model. Following Wang et al., Zhu et al. in [51] developed a PDE mathematical modeling with 
consideration of a delayed feedback controller to effectively control the diffusion of harmful information in online social networks. To our knowledge, considering PDE rumor propagation model in online social networks is still at the preliminary stage. Therefore, these spatial-temporal models will provide a new insight to for studying rumor propagation in online social networks.

It is worth noting that most of the above-mentioned works on rumor propagation modeling assume that there is no time delay over rumor propagation. In fact, in emergency situations, a government sometimes cannot promptly take notice of the perniciousness of rumors [51]. Thus, the interplay between rumor diffusion and authorities' actions in an emergency situation is not always synchronized. That is, we should assume that a time delay exists before the authorities' actions on rumor diffusion. Further, as in epidemic models [15, 26, 52], when studying the process of rumor propagation we should consider that there exists an incubation period before an influenced ignorant user can or will spread rumors. In this paper, our fundamental purpose is to present a novel rumor propagation model with more realistic significance in theory and further analyse the dynamic characteristic of this model in mathematics. Our main contributions and advances are summarized as follows.

(i) Improvements on the previous rumor propagation models. The deterministic epidemiclike rumor propagation models proposed for online social networks in the majority of existing literatures are mainly based on ODE [24, 30, 35, 42, 45], which deals only with collective social processes over time without considering space factors for mobile online social networks. Though some researchers recently have begun to study both temporal and spatial patterns of information diffusion in social media by PDE [31, 32, 51], they may ignore the spatial-temporal delay phenomenon in information transmission. In our work, based on the theory of partial function differential equations (PFDEs), we develop a spatial-temporal epidemic-like rumor propagation model with the discrete and nonlocal delays, which makes up perfectly for the deficiencies of the previous literatures.

(ii) Innovations about parameter identification of a rumor propagation model. As is well known, many factors, such as the spreading rate, the recovery rate, the forgetting rate, the average degree of the network and so on, may affect the evolution of a dynamical process. Thus, distinguishing the sensitivity of these parameters is significant for understanding the mechanism of rumor propagation in online social networks. However, in most of the previous literatures, scholars randomly selected a parameter and then varied the values of this parameter to give a numerical simulation about their proposed models [23, 24, 44, 45]. In this work, by applying sensitivity analysis in mathematics, we study the relationship between the density of spreading users and the parameters in our proposed model. This provides a new insight whith which to research rumor propagation and makes up for the deficiencies in the selection of parameters for the previous literatures.

(iii) Investigation of the function of the spatial-temporal delay in the rumor propagation model. Though previous studies $[31,32,51]$ have developed a PDE mathematical model to describe the rumor propagation mechanism, they do not concretely reveal and interpret the impact of the spatial-temporal delay on rumor propagation. In our work, by numerical simulation, we have successfully analyzed the effect of the spatial-temporal delay in two aspects. First, when the rumor propagation model is stable, the spatial-temporal delay can affect the convergence time of the model; and second, by adjusting the spatial-temporal delay, an unstable positive equilibrium point of the model can become stable. 
The remainder of this paper is organized as follows. In Section 2, the modeling approach is described explicitly. In Section 3, we study the local stability and the existence of Hopf bifurcation for our proposed model. In Section 4, to support our theoretical predictions, some numerical simulations are given. Finally, a brief discussion is given to conclude this work.

\section{Modeling Rumor Propagation}

This section describes a spatial-temporal rumor propagation model with discrete and nonlocal delays. Our goal is trying to create a realistic model that can provide broad insight into predicting and controlling rumor prevalence in online social networks.

Generally, an online social network consists of many mobile Internet users. The geographic position of a user is represented by the distance $x$ from the rumor source [31]. At any time, a user is classified as either internal or external according to whether or not he or she is connected to the networks at that time. Based on the classical SI epidemic model, in this work, the users in an online social network can be divided into two classes depending on their different states: ignorant (i.e., a user who lacks social and self-protection awareness may be infected by the rumor propagation) and spreading (i.e., a user is infected by rumor, meaning the user may infect his neighbors by spreading the rumor, but the rumor has not been initiated by the user). For simplicity, we use $I(t, x)$ and $S(t, x)$ to represent the densities of ignorant users and spreading users with a distance of $x$ at time $t$, respectively.

To model the propagation of rumor throughout online social networks, the following assumptions are imposed

(i) As previous studies mentioned [43, 44, 45], a general social network is not a regular network, and the number of people whom each individual directly contacts in reality usually approximatively satisfies a Poisson distribution. Therefore, in this paper, we consider the online social network as a homogenous network, composed of a closed and homogeneously mixed population. That is to say, in our present work, rumor propagation between each individual is restricted on a homogenous network.

(ii) In accordance with $[37,41,48]$, we consider that ignorant users usually have logistic growth with a carrying capacity as well as an intrinsic growth rate.

(iii) In online social networks, when an ignorant user is infected by spreading users, there is a spreading incubation period during which the infectious agents develop on networks, and it is only after that time that the infected users become themselves infectious. Further, due to the users' mobility, defining a spatio-temporal delay or nonlocal delay for the spreading incubation period is more appropriate [5].

(iv) Usually, when a government responds to the rumor propagation in online social networks, a time delay exists, during which the government takes effective action to control and remove the spreading users $[50,51]$.

(vi) Sometimes, rumor diffusion is different from epidemic diffusion as spreading users may forget or decline to spread the rumor $[44,45]$. Obviously, as time passes, forgetting or use of a 
stifling mechanism may result in the cessation of rumor propagation.

From the above description, our model can be represented as a set of coupled PFDEs as follows.

$$
\left\{\begin{array}{l}
\frac{\partial I(t, x)}{\partial t}=d \frac{\partial^{2} I(t, x)}{\partial x^{2}}+r I(t, x)\left(1-\frac{I(t, x)}{K}\right)-\bar{k} \beta I(t, x) \int_{\Omega} \int_{-\infty}^{t} G(t-\xi, x, y) f(t-\xi) S(\xi, y) d \xi d y \\
\frac{\partial S(t, x)}{\partial t}=d \frac{\partial^{2} S(t, x)}{\partial x^{2}}+\bar{k} \beta I(t, x) \int_{\Omega} \int_{-\infty}^{t} G(t-\xi, x, y) f(t-\xi) S(\xi, y) d \xi d y-\eta S(t-\tau, x)-\mu S(t, x),
\end{array}\right.
$$

for $t>0, x \in \Omega$ with homogeneous Neumann boundary conditions

$$
\frac{\partial I}{\partial v}(t, x)=\frac{\partial S}{\partial v}(t, x)=0, \quad t \geq 0, x \in \partial \Omega,
$$

and initial conditions

$$
\left\{\begin{array}{l}
I(t, x)=I_{0}(t, x) \geq 0,(t, x) \in(-\infty, 0] \times \bar{\Omega} \\
S(t, x)=S_{0}(t, x) \geq 0,(t, x) \in(-\infty, 0] \times \bar{\Omega}
\end{array}\right.
$$

where

$$
G(t, x, y)=\frac{1}{\pi}+\frac{2}{\pi} \sum_{n=0}^{\infty} e^{-d n^{2} t} \cos n x \cos n y, \quad f(t)=\frac{1}{T} e^{-\frac{t}{T}}, \quad \Omega=[0, \pi],
$$

$G(x, y, t)$ is the weighting function accounting for the drift of individuals to their present position (at time $t$ ) from all possible positions at all previous times [36] and essentially satisfies the heat equation $[5,29]$ :

$$
\int_{\Omega} G(t, x, y) d y=1, \frac{\partial G}{\partial t}-d \frac{\partial^{2} G}{\partial y^{2}}=0, G(0, x, y)=\delta(x, y) ;
$$

the function $f(t)$, which is called the delay kernel $[5,29]$, is usually used as a mathematical tool to weight the distributed time-delay and satisfies $f(t) \geq 0$ for all $t \geq 0$ together with the normalisation condition

$$
\int_{0}^{\infty} f(t) d t=1
$$

In system (2.1) (2.3), $d \frac{\partial^{2}}{\partial x^{2}}$ is a diffusion term, being used to describe the impact of the mobility of users at a distance of $x$. The term $\int_{\Omega} \int_{-\infty}^{t} G(t-\xi, x, y) f(t-\xi) S(\xi, y) d \xi d y$ represents the nonlocal delay due to the spreading incubation period, that is, it is only after that spatio-temporal delay that the infected users become themselves infectious, and then they can spread rumors in online social networks. The boundary condition in (2.2) implies that there are no rumors across the boundary of $\Omega$. $I_{0}(t, x)$ and $S_{0}(t, x)$ are the initial density functions. They are non-negative, Hölder continuous and satisfy $\partial I_{0} / \partial v=0$ and $\partial S_{0} / \partial v=0$ on $(-\infty, 0] \times \partial \Omega$. For clarity, we list the meanings of the other positive parameters in Table 1. 


\begin{tabular}{cl}
\hline Parameters & Notes \\
\hline $\mathrm{d}$ & The diffusion coefficient of users, being used to describe the mobility of users \\
$\mathrm{r}$ & The intrinsic growth rate of ignorant users \\
$\bar{k}$ & The average degree of the network, being used to describe the network structure \\
$\beta$ & The infection rate of an ignorant user \\
$\eta$ & The control rate of the authorities' actions in rumor propagation \\
$\mu$ & The forgetting rate of spreading users \\
$T$ & The spatial-temporal delay during which the infected users develop into the real spreading users \\
$\tau$ & The time delay during which the government responses to rumor propagation \\
$\mathrm{K}$ & The carrying capacity of online social networks \\
$\Omega$ & The bounded domain with smooth boundary $\partial \Omega$ \\
$v$ & The outward normal vector on the smooth boundary $\partial \Omega$ \\
\hline
\end{tabular}

\section{Local Stability and Hopf Bifurcation}

In this section, by analyzing the corresponding characteristic equations, we will discuss the local stability and Hopf bifurcation of system (2.1) with the time delay $\tau$ as the bifurcation parameter.

For simplicity, at the beginning, we let

$$
u=\int_{\Omega} \int_{-\infty}^{t} G(t-s, x, y) f(t-s) S(s, y) d s d y, \quad \alpha=\frac{1}{T},
$$

then system (2.1) can be rewritten as

$$
\left\{\begin{array}{l}
\frac{\partial I}{\partial t}=d \frac{\partial^{2} I}{\partial x^{2}}+r I\left(1-\frac{I}{K}\right)-\bar{k} \beta I u \\
\frac{\partial S}{\partial t}=d \frac{\partial^{2} S}{\partial x^{2}}+\bar{k} \beta I u-\mu S-\eta S(t-\tau) \\
\frac{\partial u}{\partial t}=d \frac{\partial^{2} u}{\partial x^{2}}+\alpha(S-u)
\end{array}\right.
$$

It can be seen that homogeneous Neumann boundary conditions imposed on (3.1) lead to $E^{0}=(0,0,0)^{T}$ being the trivial equilibrium point for any feasible parameters, $E^{1}=(K, 0,0)^{T}$ always being the boundary equilibrium point, and system (3.1) always having a unique positive equilibrium point $E^{*}=\left(I^{*}, S^{*}, u^{*}\right)^{T}$ provided that the condition $\left(H_{1}\right) K \bar{k} \beta-\mu-\eta>0$ holds, where

$$
I^{*}=\frac{\eta+\mu}{\bar{k} \beta}, \quad S^{*}=\frac{r(K \bar{k} \beta-\mu-\eta)}{K \bar{k}^{2} \beta^{2}}, \quad u^{*}=\frac{r(K \bar{k} \beta-\mu-\eta)}{K \bar{k}^{2} \beta^{2}} .
$$

Let $\tilde{I}=I-I^{\star}, \tilde{S}=S-S^{\star}, \tilde{u}=u-u^{\star}$, where $\left(I^{\star}, S^{\star}, u^{\star}\right)^{T}$ is an arbitrary equilibrium point, and drop bars for simplicity. Then system (3.1) can be transformed into the following form 


$$
\left\{\begin{array}{l}
\frac{\partial I}{\partial t}=d \frac{\partial^{2} I}{\partial x^{2}}+\left(r-\frac{2 r I^{\star}}{K}-\bar{k} \beta u^{\star}\right) I-\bar{k} \beta I^{\star} u-\frac{r}{K} I^{2}-\bar{k} \beta I u, \\
\frac{\partial S}{\partial t}=d \frac{\partial^{2} S}{\partial x^{2}}+\bar{k} \beta u^{\star} I-\mu S-\eta S(t-\tau)+\bar{k} \beta I^{\star} u+\bar{k} \beta I u, \\
\frac{\partial u}{\partial t}=d \frac{\partial^{2} u}{\partial x^{2}}+\alpha(S-u) .
\end{array}\right.
$$

Thus, the arbitrary equilibrium point $E^{\star}=\left(I^{\star}, S^{\star}, u^{\star}\right)^{T}$ of system (3.1) is transformed into the zero equilibrium point $(0,0,0)^{T}$ of system (3.2).

In the following, we will analyze stability and bifurcation of the zero equilibrium point of system (3.2).

Denote

$$
U(t)=\left(u_{1}(t), u_{2}(t), u_{3}(t)\right)^{T}=(I(t, \cdot), S(t, \cdot), u(t, \cdot))^{T}
$$

then system (3.2) can be rewritten as an abstract differential equation in the phase space $C=$ $C([-\tau, 0], X)$ of the form

$$
\dot{U}=D \Delta U(t)+L\left(U_{t}\right)+f\left(U_{t}\right),
$$

where

$D=\operatorname{diag}\{d, d, d\}$,

$\Delta=\operatorname{diag}\left\{\partial^{2} / \partial x^{2}, \partial^{2} / \partial x^{2}, \partial^{2} / \partial x^{2}\right\}$,

$U_{t}(\theta)=U(t+\theta),-\tau \leq \theta \leq 0$,

$L: C \rightarrow X$

and

$f: C \rightarrow X$ are given, respectively, by

$$
L(\varphi)=\left(\begin{array}{c}
\left(r-\bar{k} \beta u^{\star}-\frac{2 r I^{\star}}{K}\right) \varphi_{1}(0)-\bar{k} \beta I^{\star} \varphi_{3}(0) \\
\bar{k} \beta u^{\star} \varphi_{1}(0)-\mu \varphi_{2}(0)-\eta \varphi_{2}(-\tau)+\bar{k} \beta I^{\star} \varphi_{3}(0) \\
\alpha \varphi_{2}(0)-\alpha \varphi_{3}(0)
\end{array}\right)
$$

and

$$
f(\varphi)=\left(\begin{array}{c}
-\frac{r}{K} \varphi_{1}^{2}(0)-\bar{k} \beta \varphi_{1}(0) \varphi_{3}(0) \\
\bar{k} \beta \varphi_{1}(0) \varphi_{3}(0) \\
0
\end{array}\right)
$$

For $\varphi(\theta)=U_{t}(\theta), \varphi=\left(\varphi_{1}, \varphi_{2}, \varphi_{3}\right)^{T} \in C$, the linearized system of (3.3) at the zero equilibrium point is

$$
\dot{U}=D \Delta U(t)+L\left(U_{t}\right)
$$

and its characteristic equation is

$$
\lambda w-D \Delta w-L\left(e^{\lambda \cdot} w\right)=0,
$$

where $w \in \operatorname{dom}(\Delta)$, and $w \neq 0, \operatorname{dom}(\Delta) \subset X$. 
From the properties of the Laplacian operator defined on the bounded domain, the operator $\Delta$ on $X$ has the eigenvalues $-m^{2}, m \in N_{0} \triangleq\{0,1,2 \cdots\}$ with the relative eigenfunctions $\beta_{m}^{1}, \beta_{m}^{2}, \beta_{m}^{3}$, where

$$
\beta_{m}^{1}=\left(\begin{array}{l}
\gamma_{m} \\
0 \\
0
\end{array}\right), \beta_{m}^{2}=\left(\begin{array}{l}
0 \\
\gamma_{m} \\
0
\end{array}\right), \beta_{m}^{3}=\left(\begin{array}{l}
0 \\
0 \\
\gamma_{m}
\end{array}\right), \gamma_{m}=\cos (m x) .
$$

Clearly, $\left(\beta_{m}^{1}, \beta_{m}^{2}, \beta_{m}^{3}\right)_{0}^{\infty}$ is a basis of the phase space $X$. Therefore, any element $w$ in $X$ can be expanded as Fourier series in the following form

$$
w=\sum_{m=0}^{\infty} W_{m}^{T}\left(\begin{array}{c}
\beta_{m}^{1} \\
\beta_{m}^{2} \\
\beta_{m}^{3}
\end{array}\right), W_{m}=\left(\begin{array}{c}
<w, \beta_{m}^{1}> \\
<w, \beta_{m}^{2}> \\
<w, \beta_{m}^{3}>
\end{array}\right) .
$$

By calculation

$$
L\left(\varphi^{T}\left(\beta_{m}^{1}, \beta_{m}^{2}, \beta_{m}^{3}\right)^{T}\right)=L^{T}(\varphi)\left(\beta_{m}^{1}, \beta_{m}^{2}, \beta_{m}^{3}\right), \quad m \in N_{0} .
$$

According to (3.8) and (3.9), (3.7) is equivalent to

$$
\sum_{m=0}^{\infty} W_{m}^{T}\left[\lambda I_{3}+D m^{2}-\left(\begin{array}{ccc}
r-\bar{k} \beta u^{\star}-\frac{2 r I^{\star}}{K} & 0 & -\bar{k} \beta I^{\star} \\
\bar{k} \beta u^{\star} & -\mu-\eta e^{-\lambda \tau} & \bar{k} \beta I^{\star} \\
0 & \alpha & -\alpha
\end{array}\right)\right]\left(\begin{array}{c}
\beta_{m}^{1} \\
\beta_{m}^{2} \\
\beta_{m}^{3}
\end{array}\right)=0 .
$$

Thus the characteristic equation is

$$
\lambda^{3}+A_{1} \lambda^{2}+A_{2} \lambda+A_{3}+\left(\lambda^{2}+A_{4} \lambda+A_{5}\right) \eta e^{-\lambda \tau}=0,
$$

where

$$
\begin{aligned}
A_{1}= & 3 d m^{2}+\mu+\alpha+\bar{k} \beta u^{\star}+\frac{2 r}{K} I^{\star}-r, \\
A_{2}= & 3 d^{2} m^{4}+2\left(\mu+\alpha+\bar{k} \beta u^{\star}+\frac{2 r}{K} I^{\star}-r\right) d m^{2}+(\alpha+\mu)\left(\bar{k} \beta u^{\star}+\frac{2 r}{K} I^{\star}-r\right)+\alpha \mu-\bar{k} \beta \alpha I^{\star}, \\
A_{3}= & d^{3} m^{6}+\left(\mu+\alpha+\bar{k} \beta u^{\star}+\frac{2 r}{K} I^{\star}-r\right) d^{2} m^{4}+\left[(\alpha+\mu)\left(\bar{k} \beta u^{\star}+\frac{2 r}{K} I^{\star}-r\right)+\alpha \mu-\bar{k} \beta \alpha I^{\star}\right] d m^{2} \\
& +\bar{k}^{2} \beta^{2} \alpha I^{\star} u^{\star}+\left(\alpha \mu-\bar{k} \beta \alpha I^{\star}\right)\left(\bar{k} \beta u^{\star}+\frac{2 r}{K} I^{\star}-r\right), \\
A_{4}= & 2 d m^{2}+\alpha+\bar{k} \beta u^{\star}+\frac{2 r}{K} I^{\star}-r, \\
A_{5}= & d^{2} m^{4}+\left(\alpha+\bar{k} \beta u^{\star}+\frac{2 r}{K} I^{\star}-r\right) d m^{2}+\alpha\left(\bar{k} \beta u^{\star}+\frac{2 r}{K} I^{\star}-r\right) .
\end{aligned}
$$

\subsection{Stability and Hopf bifurcation of boundary equilibrium point $E^{1}$}

According to the above analysis, in this part, we take

$$
\left(I^{\star}, S^{\star}, u^{\star}\right)^{T}=E^{1}=(K, 0,0)^{T} .
$$


Thus, the characteristic equation (3.12) is transformed into the following form

$$
\begin{aligned}
\lambda^{3}+\left(3 d m^{2}+\right. & \left.B_{1}\right) \lambda^{2}+\left(3 d^{2} m^{4}+2 B_{1} d m^{2}+B_{2}\right) \lambda+\left(d^{3} m^{6}+B_{1} d^{2} m^{4}+B_{2} d m^{2}+B_{3}\right) \\
& +\left[\lambda^{2}+\left(2 d m^{2}+B_{4}\right) \lambda+d^{2} m^{4}+B_{4} d m^{2}+B_{5}\right] \eta e^{-\lambda \tau}=0,
\end{aligned}
$$

where

$$
B_{1}=\alpha+\mu+r, B_{2}=\alpha \mu+\alpha r+\mu r-\bar{k} \alpha \beta K, B_{3}=\alpha r(\mu-\bar{k} \beta K), B_{4}=\alpha+r, B_{5}=\alpha r .
$$

Further, make the following assumptions

$\left(H_{11}\right) \quad \mu+\eta-\bar{k} \beta K>0$

( $\left.H_{12}\right) \quad \mu-\eta-\bar{k} \beta K>0$

$\left(H_{13}\right) \quad \alpha(\mu-\bar{k} \beta K)(\alpha+\mu-2 r)+(\alpha+r)\left(\alpha r+\mu r-\eta^{2}\right)>0$

Lemma 1. If $\left(H_{11}\right)$ holds, then system (3.1) is locally asymptotically stable at the boundary equilibrium point $E^{1}$ as $\tau=0$.

Proof. Obviously, for $\forall m \in N_{0}, \lambda=0$ is not a root of Eq.(3.13). As $\tau=0$, Eq.(3.13) is equivalent to the following cubic equation

$\lambda^{3}+\left(3 d m^{2}+B_{1}+\eta\right) \lambda^{2}+\left[3 d^{2} m^{4}+2\left(B_{1}+\eta\right) d m^{2}+B_{2}+B_{4} \eta\right] \lambda+\left[d^{3} m^{6}+\left(B_{1}+\eta\right) d^{2} m^{4}+\left(B_{2}+B_{4} \eta\right) d m^{2}+B_{3}+B_{5} \eta\right]=0$.

Clearly, if $\left(H_{11}\right)$ holds, we get

$$
\begin{aligned}
& B_{2}+B_{4} \eta=\alpha(\mu+\eta-\bar{k} \beta K)+r(\mu+\alpha+\eta)>0, \\
& B_{3}+B_{5} \eta=\alpha r(\mu+\eta-\bar{k} \beta K)>0, \\
& \left(3 d m^{2}+B_{1}+\eta\right)\left[3 d^{2} m^{4}+2\left(B_{1}+\eta\right) d m^{2}+B_{2}+B_{4} \eta\right]-\left[d^{3} m^{6}+\left(B_{1}+\eta\right) d^{2} m^{4}+\left(B_{2}+B_{4} \eta\right) d m^{2}+B_{3}+B_{5} \eta\right] \\
& =8 d^{3} m^{6}+8\left(B_{1}+\eta\right) d^{2} m^{4}+2\left(B_{2}+B_{4} \eta\right) d m^{2}+2\left(B_{1}+\eta\right)^{2} d m^{2}+\left(B_{2}+B_{4} \eta+r^{2}\right)(\mu+\eta+\alpha)>0 .
\end{aligned}
$$

According to the Routh-Hurwitz criteria, all the roots of Eq.(3.14) have negative real parts. Therefore, when $\tau=0$, the boundary equilibrium point $E^{1}$ is locally asymptotically stable.

Theorem 1. If $\left(H_{12}\right) \sim\left(H_{13}\right)$ hold, then the boundary equilibrium point $E^{1}$ of system (3.1) is locally asymptotically stable for $\forall \tau \geq 0$.

Proof. Obviously, if $\left(H_{12}\right)$ satisfies, then Lemma 1 holds. That is, when $\tau=0$ system (3.1) is locally asymptotically stable at the boundary equilibrium point $E^{1}$. Next we discuss the effect of the delay $\tau$ on the stability of the trivial solution of Eq.(3.13). Assume that $i \omega$ is a root of Eq.(3.13). Then $\omega$ should satisfy the following equation for $m \in N_{0}$

$$
\begin{gathered}
-i \omega^{3}-\omega^{2}\left(3 d m^{2}+B_{1}\right)+i \omega\left(3 d^{2} m^{4}+2 B_{1} d m^{2}+B_{2}\right)+\left(d^{3} m^{6}+B_{1} d^{2} m^{4}+B_{2} d m^{2}+B_{3}\right) \\
+\left[-\omega^{2}+i \omega\left(2 d m^{2}+B_{4}\right)+d^{2} m^{4}+B_{4} d m^{2}+B_{5}\right] \eta e^{-i \omega \tau}=0,
\end{gathered}
$$

which implies that

$$
\left\{\begin{aligned}
\left(-\omega^{2}+d^{2} m^{4}+B_{4} d m^{2}+B_{5}\right) \eta \cos \omega \tau+\left(2 d m^{2}+B_{4}\right) \eta \omega \sin \omega \tau & =\left(3 d m^{2}+B_{1}\right) \omega^{2} \\
& -\left(d^{3} m^{6}+B_{1} d^{2} m^{4}+B_{2} d m^{2}+B_{3}\right), \\
\left(2 d m^{2}+B_{4}\right) \eta \omega \sin \omega \tau-\left(-\omega^{2}+d^{2} m^{4}+B_{4} d m^{2}+B_{5}\right) \eta \cos \omega \tau & =\omega^{3}-\omega\left(3 d^{2} m^{4}+2 B_{1} d m^{2}+B_{2}\right) .
\end{aligned}\right.
$$


Taking square on both sides of the equations of (3.16) and summing them up, we obtain

$$
\omega^{6}+C_{1 m} \omega^{4}+C_{2 m} \omega^{2}+C_{3 m}=0
$$

where

$$
\begin{aligned}
C_{1 m}= & 3 d^{2} m^{4}+2 B_{1} d m^{2}+\left(\alpha^{2}+r^{2}+\mu^{2}-\eta^{2}+2 \bar{k} \beta \alpha K\right), \\
C_{2 m}= & 3 d^{4} m^{8}+4 B_{1} d^{3} m^{6}+2\left[(\alpha+r+\mu)^{2}-\eta^{2}\right] d^{2} m^{4} \\
& +2\left(B_{1} B_{2}-B_{4} \eta^{2}-3 B_{3}\right) d m^{2}+B_{2}^{2}-\eta^{2}\left(\alpha^{2}+r^{2}\right)-2 B_{1} B_{3}, \\
C_{3 m}= & \left(d^{3} m^{6}+B_{1} d^{2} m^{4}+B_{2} d m^{2}+B_{3}\right)^{2}-\eta^{2}\left(d^{2} m^{4}+B_{4} d m^{2}+B_{5}\right)^{2} .
\end{aligned}
$$

Set $z=\omega^{2}$, Eq.(3.17) is transformed into the following equation

$$
z^{3}+C_{1 m} z^{2}+C_{2 m} z+C_{3 m}=0 .
$$

Clearly, when $\left(H_{12}\right) \sim\left(H_{13}\right)$ hold, it is easy to show that

$$
\begin{aligned}
C_{1 m}= & 3 d^{2} m^{4}+2 B_{1} d m^{2}+(\mu-\eta)(\mu+\eta)+\alpha^{2}+r^{2}+2 \bar{k} \beta \alpha K>0, \\
C_{2 m}= & 3 d^{4} m^{8}+4 B_{1} d^{3} m^{6}+2[r(2 \alpha+2 \mu+r)+(\alpha+\mu-\eta)(\alpha+\mu+\eta)] d^{2} m^{4} \\
& +2\left[\alpha(\mu-\bar{k} \beta K)(\alpha+\mu-2 r)+(\alpha+r)\left(\alpha r+\mu r-\eta^{2}\right)+2 \mu r(\alpha+\mu)\right] d m^{2} \\
& +\left[\alpha^{2}(\mu+\eta-\bar{k} \beta K)(\mu-\eta-\bar{k} \beta K)+r^{2} \alpha(\alpha+2 \bar{k} \beta K)+r^{2}(\mu-\eta)(\mu+\eta)\right]>0, \\
C_{3 m}= & \left\{d^{3} m^{6}+(\alpha+\mu+r+\eta) d^{2} m^{4}+[\alpha(\mu+\eta-\bar{k} \beta K)+r(\alpha+\mu+\eta)] d m^{2}+\alpha r(\mu+\eta-\bar{k} \beta K)\right\} \\
& \times\left\{d^{3} m^{6}+(\alpha+\mu+r-\eta) d^{2} m^{4}+[\alpha(\mu-\eta-\bar{k} \beta K)+r(\alpha+\mu-\eta)] d m^{2}+\alpha r(\mu-\eta-\bar{k} \beta K)\right\}>0 .
\end{aligned}
$$

These inequations imply that Eq.(3.18) has no positive roots, and hence Eq. (3.13) has no purely imaginary roots. Combing with Lemma 1, all roots of (3.13) have negative real parts as $\tau \geq 0$. Thus, $E^{1}$ is locally asymptotically stable for $\forall \tau \geq 0$. This completes the proof.

Now we further discuss how Hopf bifurcation of the boundary equilibrium point $E^{1}$ is created by the delay $\tau$. For further discussion, we assume

$$
\begin{array}{ll}
\left(H_{14}\right) & \mu-\eta-\bar{k} \beta K<0 \\
\left(H_{15}\right) & \alpha-\eta \geq 0 \\
\left(H_{16}\right) & r^{2}\left(\alpha^{2}+\mu^{2}+2 \bar{k} \beta \alpha K\right)-\eta^{2}\left(\alpha^{2}+r^{2}\right)>0 \\
\left(H_{17}\right) & \frac{d^{2}}{2}+(\mu-\eta)(\alpha+r)+\alpha(r-\bar{k} \beta K)>0 \\
\left(H_{18}\right) & \frac{d^{3}}{2}+\alpha r(\mu-\eta-\bar{k} \beta K)>0
\end{array}
$$

Lemma 2. When $m=0$, Eq. (3.18) has a unique positive real root provided that the conditions $H_{11} H_{14}$ and $H_{15}$ hold.

Proof. Denote

$$
g(z)=z^{3}+C_{10} z^{2}+C_{20} z+C_{30} .
$$


Since $g(0)=\alpha^{2} r^{2}(\mu+\eta-\bar{k} \beta K)(\mu-\eta-\bar{k} \beta K)<0, \lim _{z \rightarrow \infty} g(z)=\infty$. Thus, it is obvious that Eq.(3.18) has at least one positive root. Moreover, under the condition $\left(H_{15}\right)$ and according to Descartes's rule of signs, we have $C_{10}>0$. Thus, Eq.(3.18) has a unique positive root. This completes the proof.

Without loss of generality, we defined the unique positive real root of Eq.(3.18) by $z_{1}$. Then we have $\omega_{1}=\sqrt{z_{1}}$.

By (3.16), we obtain

$$
\cos \omega_{1} \tau_{1}=\frac{-\mu \omega_{1}^{4}+\left(\bar{k} \beta K \alpha^{2}-\mu \alpha^{2}-\mu r^{2}\right) \omega_{1}^{2}+\alpha^{2} r^{2}(\bar{k} \beta K-\mu)}{\eta\left[\omega_{1}^{4}+\left(\alpha^{2}+r^{2}\right) \omega_{1}^{2}+\alpha^{2} r^{2}\right]},
$$

thus, if we denote

$$
\tau_{1}^{j}=\frac{1}{\omega_{1}} \arccos \left(\frac{-\mu \omega_{1}^{4}+\left(\bar{k} \beta K \alpha^{2}-\mu \alpha^{2}-\mu r^{2}\right) \omega_{1}^{2}+\alpha^{2} r^{2}(\bar{k} \beta K-\mu)}{\eta\left[\omega_{1}^{4}+\left(\alpha^{2}+r^{2}\right) \omega_{1}^{2}+\alpha^{2} r^{2}\right]}+2 j \pi\right),
$$

where $j=0,1,2, \ldots$, then $\pm i \omega_{1}$ is a pair of purely imaginary roots of (3.13) with $\tau_{1}^{j}$. Clearly, sequence $\left\{\tau_{1}^{j}\right\}_{j=0}^{\infty}$ is increasing and $\lim _{j \rightarrow+\infty} \tau_{1}^{j}=+\infty$.

Thus, we can define

$$
\tau_{0}^{*}=\tau_{1}^{0}=\min \left\{\tau_{1}^{j}\right\}
$$

Lemma 3. If $\left(H_{11}\right)$ and $\left(H_{13}\right)$ hold. Assume furthermore that $\left(H_{15}\right) \sim\left(H_{18}\right)$ satisfy. Then, for $\forall m \geq 1$, Eq.(3.18) has no positive real roots.

Proof. Obviously, from $\left(H_{15}\right)$, we have

$$
C_{1 m}=3 d^{2} m^{4}+2 B_{1} d m^{2}+\mu^{2}+(\alpha-\eta)(\alpha+\eta)+r^{2}+2 \bar{k} \beta \alpha K>0 .
$$

As $\left(H_{13}\right)$ and $\left(H_{15}\right) \sim\left(H_{16}\right)$ hold, we can obtain

$$
\begin{aligned}
C_{2 m}= & 3 d^{4} m^{8}+4 B_{1} d^{3} m^{6}+2[r(2 \alpha+2 \mu+r)+(\alpha+\mu-\eta)(\alpha+\mu+\eta)] d^{2} m^{4} \\
& +2\left[\alpha(\mu-\bar{k} \beta K)(\alpha+\mu-2 r)+(\alpha+r)\left(\alpha r+\mu r-\eta^{2}\right)+2 \mu r(\alpha+\mu)\right] d m^{2} \\
& +\alpha^{2}(\mu-\bar{k} \beta K)^{2}+\left[r^{2}\left(\alpha^{2}+\mu^{2}+2 \bar{k} \beta \alpha K\right)-\eta^{2}\left(\alpha^{2}+r^{2}\right)\right]>0 .
\end{aligned}
$$

According to $\left(H_{11}\right)\left(H_{15}\right)$ and $\left(H_{17}\right) \sim\left(H_{18}\right)$, a simple calculation shows that

$$
\begin{aligned}
C_{3 m} \geq & \left\{d^{3} m^{6}+(\alpha+\mu+r+\eta) d^{2} m^{4}+[\alpha(\mu+\eta-\bar{k} \beta K)+r(\alpha+\mu+\eta)] d m^{2}+\alpha r(\mu+\eta-\bar{k} \beta K)\right\} \\
& \times\left\{d^{2} m^{4}(\alpha+\mu+r-\eta)+d m^{2}\left[\frac{d^{2}}{2}+(\mu-\eta)(\alpha+r)+\alpha(r-\bar{k} \beta K)\right]+\left[\frac{d^{3}}{2}+\alpha r(\mu-\eta-\bar{k} \beta K)\right]\right\}>0 .
\end{aligned}
$$

These inequations imply that Eq.(3.18) has no positive roots for $\forall m \geq 1$. 
Lemma 4. Let $\lambda(\tau)=\gamma(\tau) \pm i \omega(\tau)$ be the root of (3.13) near $\tau=\tau_{0}^{*}$ satisfying $\gamma\left(\tau_{0}^{*}\right)=0$, $\omega\left(\tau_{0}^{*}\right)=\omega_{1}$. Suppose that $\left(H_{15}\right) \sim\left(H_{16}\right)$ exist. Then, the following transversality condition holds

$$
\left.\frac{d(\operatorname{Re} \lambda(\tau))}{d \tau}\right|_{\tau=\tau_{0}^{*}, \lambda=i \omega_{1}}>0
$$

Proof. When $m=0$, differentiating the two sides of (3.13) with respect to $\tau$ yields

$$
\left(\frac{d \lambda}{d \tau}\right)^{-1}=\frac{\left(3 \lambda^{2}+2 B_{1} \lambda+B_{2}\right) e^{\lambda \tau}+\eta\left(2 \lambda+B_{4}\right)}{\eta \lambda\left(\lambda^{2}+B_{4} \lambda+B_{5}\right)}-\frac{\tau}{\lambda} .
$$

Therefore, according to $\left(H_{15}\right) \sim\left(H_{16}\right)$, we can easily obtain

$$
\begin{aligned}
& {\left[\frac{d(\operatorname{Re} \lambda(\tau))}{d \tau}\right]_{\tau=\tau_{0}^{*}, \lambda=i \omega_{1}}^{-1}=\operatorname{Re}\left[\frac{\left(3 \lambda^{2}+2 B_{1} \lambda+B_{2}\right) e^{\lambda \tau}+\eta\left(2 \lambda+B_{4}\right)}{\eta \lambda\left(\lambda^{2}+B_{4} \lambda+B_{5}\right)}\right]_{\tau=\tau_{0}^{*}, \lambda=i \omega_{1}}} \\
& =\frac{\left[3 \omega_{1}^{4}+\left(2 B_{1} B_{4}-B_{2}-3 B_{5}\right) \omega_{1}^{2}+B_{2} B_{5}\right] \sin \omega_{1} \tau_{1}^{*}+\left[\left(3 B_{4}-2 B_{1}\right) \omega_{1}^{3}+\left(2 B_{1} B_{5}-B_{2} B_{4}\right) \omega_{1}\right] \cos \omega_{1} \tau_{1}^{*}}{\eta \omega_{1}\left[B_{4}^{2} \omega_{1}^{2}+\left(\omega_{1}^{2}-B_{5}\right)^{2}\right]} \\
& =\frac{3 \omega_{1}^{4}+2\left[(\alpha-\eta)(\alpha+\eta)+\mu^{2}+r^{2}+2 \bar{k} \beta \alpha K\right] \omega_{1}^{2}+\alpha^{2}(\mu-\bar{k} \beta K)^{2}+\left[r^{2}\left(\alpha^{2}+\mu^{2}+2 \bar{k} \beta \alpha K\right)-\eta^{2}\left(\alpha^{2}+r^{2}\right)\right]}{\eta^{2}\left[B_{4}^{2} \omega_{1}^{2}+\left(\omega_{1}^{2}-B_{5}\right)^{2}\right]} \\
& >0 .
\end{aligned}
$$

This completes the proof.

In view of Lemmas 1-4, we have the following result.

Theorem 2. Let $\tau_{0}^{*}$ be defined by (3.21). If $\left(H_{11}\right)$ and $\left(H_{13}\right) \sim\left(H_{18}\right)$ hold, then the following statements are true.

(i) When $\tau \in\left[0, \tau_{0}^{*}\right.$ ), the boundary equilibrium point $E^{1}$ of system (3.1) is locally asymptotically stable;

(ii) The Hopf bifurcation occurs at $\tau=\tau_{0}^{*}$. That is, system (3.1) has a branch of periodic solutions bifurcating from the boundary equilibrium point $E^{1}$ near $\tau=\tau_{0}^{*}$.

\subsection{Stability and Hopf bifurcation of positive equilibrium point $E^{*}$}

In this part, we will discuss the local stability and Hopf bifurcation of the positive equilibrium point $E^{*}$ with the time delay $\tau$ as the bifurcation parameter. First, we take

$$
\left(I^{\star}, S^{\star}, u^{\star}\right)^{T}=E^{*}=\left(\frac{\eta+\mu}{\bar{k} \beta}, \frac{r(K \bar{k} \beta-\mu-\eta)}{K \bar{k}^{2} \beta^{2}}, \frac{r(K \bar{k} \beta-\mu-\eta)}{K \bar{k}^{2} \beta^{2}}\right)^{T} .
$$

Thus, the characteristic equation (3.12) is transformed into the following form

$$
\lambda^{3}+Q_{1 m} \lambda^{2}+Q_{2 m} \lambda+Q_{3 m}+\left(\lambda^{2}+Q_{4 m} \lambda+Q_{5 m}\right) \eta e^{-\lambda \tau}=0,
$$


where

$$
\begin{aligned}
& Q_{1 m}=3 d m^{2}+\mu+\alpha+H, \\
& Q_{2 m}=3 d^{2} m^{4}+2(\mu+\alpha+H) d m^{2}+H \alpha+H \mu-\alpha \eta, \\
& Q_{3 m}=d^{3} m^{6}+(\mu+\alpha+H) d^{2} m^{4}+(H \mu+H \alpha-\alpha \eta) d m^{2}+\bar{k}^{2} \beta^{2} \alpha I^{\star} u^{\star}-\alpha \eta H, \\
& Q_{4 m}=2 d m^{2}+\alpha+H, \\
& Q_{5 m}=d^{2} m^{4}+(\alpha+H) d m^{2}+\alpha H, \\
& H=\frac{r(\mu+\eta)}{\bar{k} \beta K} .
\end{aligned}
$$

For further discussion, we consider the following assumptions

$\left(H_{21}\right) \quad(\alpha+\mu+\eta)^{2}-\alpha(\bar{k} \beta K-\mu-\eta)>0$

$\left(H_{22}\right) \quad \bar{k} \beta K-\mu-3 \eta<0$

$\left(H_{23}\right) \quad d^{3}-\alpha \eta(\alpha+\mu+\eta)-3 \alpha \beta \bar{k} K H>0$

$\left(H_{24}\right) \quad d^{4}+2 \alpha \eta H^{2}-2(\alpha+\mu+H) \bar{k}^{2} \beta^{2} \alpha I^{*} u^{*}>0$

$\left(H_{25}\right) \quad d^{2}>\max \{2 \alpha \eta-(\alpha+\mu-\eta) H, \alpha(\mu+3 \eta-\bar{k} K \beta)\}$

Lemma 5. If $\left(H_{21}\right)$ holds, then the positive equilibrium point $E^{*}$ of system (3.1) with $\tau=0$ is locally asymptotically stable.

Proof. Clearly, from (3.22), if $\left(H_{21}\right)$ holds, we easily obtain that

$$
\begin{aligned}
& Q_{1 m}+\eta=3 d m^{2}+\mu+\alpha+H+\eta>0, \\
& Q_{2 m}+\eta Q_{4 m}=3 d^{2} m^{4}+2(\alpha+\mu+\eta+H) d m^{2}+H(\alpha+\mu+\eta)>0, \\
& Q_{3 m}+\eta Q_{5 m}=d^{3} m^{6}+(\alpha+\mu+\eta+H) d^{2} m^{4}+(\alpha+\mu+\eta) H d m^{2}+\bar{k}^{2} \beta^{2} \alpha I^{*} u^{*}>0, \\
& \left(Q_{1 m}+\eta\right)\left(Q_{2 m}+\eta Q_{4 m}\right)-\left(Q_{3 m}+\eta Q_{5 m}\right)=8 d^{3} m^{6}+8(\alpha+\mu+H+\eta) d^{2} m^{4} \\
& +2\left[H(\alpha+\mu+\eta)+(\alpha+\mu+\eta+H)^{2}\right] d m^{2}+H\left[(\alpha+\mu+\eta)^{2}-\alpha(\bar{k} \beta K-\mu-\eta)+H(\alpha+\mu+\eta)\right]>0 .
\end{aligned}
$$

By the Routh-Hurwitz criteria, all the roots of Eq.(3.22) have negative real parts. Therefore, we have the above result.

Next we discuss the effect of the delay $\tau$ on the stability of the trivial solution of Eq.(3.22). Assume that $i \omega$ is a root of Eq.(3.22). Then $\omega$ should satisfy the following equation for $m \in N_{0}$

$$
\left\{\begin{array}{l}
\left(-\omega^{2}+Q_{5 m}\right) \eta \cos \omega \tau+Q_{4 m} \eta \omega \sin \omega \tau=Q_{1 m} \omega^{2}-Q_{3 m} \\
Q_{4 m} \eta \omega \cos \omega \tau-\left(-\omega^{2}+Q_{5 m}\right) \eta \sin \omega \tau=\omega^{3}-Q_{2 m} \omega
\end{array}\right.
$$

Taking square on both sides of the equations of (3.23) and summing them up, we obtain

$$
\omega^{6}+R_{1 m} \omega^{4}+R_{2 m} \omega^{2}+R_{3 m}=0,
$$

where $R_{1 m}=Q_{1 m}^{2}-2 Q_{2 m}-\eta^{2}, R_{2 m}=2 \eta^{2} Q_{5 m}-\eta^{2} Q_{4 m}^{2}-2 Q_{1 m} Q_{3 m}+Q_{2 m}^{2}, R_{3 m}=Q_{3 m}^{2}-\eta^{2} Q_{5 m}^{2}$. 
Set $z=\omega^{2}$, Eq.(3.24) is transformed into the following equation

$$
z^{3}+R_{1 m} z^{2}+R_{2 m} z+R_{3 m}=0 .
$$

Lemma 6. For the polynomial equation (3.25), we have the following results.

(i) If $\left(H_{15}\right)$ and $\left(H_{22}\right)$ hold, then Eq.(3.25) has a unique positive real root for $m=0$.

(ii) If $\left(H_{15}\right)$ holds. Assume furthermore that $\left(H_{23}\right) \sim\left(H_{25}\right)$ satisfy. Then Eq.(3.25) has no positive real roots for $m \geq 1$.

Proof. (i) Denote

$$
h(z)=z^{3}+R_{10} z^{2}+R_{20} z+R_{30}
$$

Similar to Lemma 2 , we can easily obtain the result when $\left(H_{15}\right)$ and $\left(H_{22}\right)$ satisfy.

(ii) Under the conditions $\left(H_{15}\right)$ and $\left(H_{23}\right) \sim\left(H_{25}\right)$, it is easy to show that

$$
\begin{aligned}
R_{1 m}= & 3 d^{2} m^{4}+2(\mu+\alpha+H) d m^{2}+(\alpha+\mu+\eta)(\alpha+\mu-\eta)+2 \alpha \eta+H^{2}>0, \\
R_{2 m} \geq & 4(\alpha+\mu+H) d^{3} m^{6}+2\left[(\alpha+\mu+\eta)(\alpha+\mu-\eta)+2 H(\alpha+\mu)+H^{2}\right] d^{2} m^{4}+\{2 H(\alpha+\mu)(\alpha \\
& \left.+\mu+H)+6 \alpha H(\mu+\eta)+2 \alpha \eta H+2 \eta H(\alpha-\eta)+2\left[d^{3}-\alpha \eta(\alpha+\mu+\eta)-3 \alpha \beta \bar{k} K H\right]\right\} d m^{2} \\
& +H^{2}(\alpha+\mu+\eta)(\alpha+\mu-\eta)+\left[d^{4}+2 \alpha \eta H^{2}-2(\alpha+\mu+H) \bar{k}^{2} \beta^{2} \alpha I^{*} u^{*}\right]>0, \\
R_{3 m} \geq & {\left[d^{3} m^{6}+(\alpha+\mu+\eta+H) d^{2} m^{4}+H(\alpha+\mu+\eta) d m^{2}+\bar{k}^{2} \beta^{2} \alpha I^{*} u^{*}\right]\left\{(\alpha+\mu-\eta) d^{2} m^{4}\right.} \\
& \left.+\left[d^{2}+H(\alpha+\mu-\eta)-2 \alpha \eta\right] d m^{2}+H\left[d^{2}+\alpha(\bar{k} K \beta-\mu-3 \eta)\right]\right\}>0 .
\end{aligned}
$$

Therefore, Eq.(3.25) has no positive roots for $\forall m \geq 1$. This completes the proof.

According to Lemma 6, if $\left(H_{15}\right)$ and $\left(H_{22}\right)$ hold, then for $m=0$ Eq.(3.25) has a unique positive root, denoted by $\bar{Z}_{1}$, and thus Eq.(3.24) has a unique positive root $\bar{\omega}_{1}=\bar{Z}_{1}$.

Combing with (3.23), we have

$$
\cos \bar{\omega}_{1} \bar{\tau}_{1}=\frac{-\mu \bar{\omega}_{1}^{4}+\left(\alpha^{2} \eta-H^{2} \mu+\bar{k}^{2} \beta^{2} \alpha I^{*} u^{*}\right) \bar{\omega}_{1}^{2}+\eta \alpha^{2} H^{2}-\bar{k}^{2} \beta^{2} \alpha^{2} H I^{*} u^{*}}{\eta\left[\bar{\omega}_{1}^{4}+\left(\alpha^{2}+H^{2}\right) \bar{\omega}_{1}^{2}+\alpha^{2} H^{2}\right]}
$$

thus, if we denote

$$
\bar{\tau}_{1}^{j}=\frac{1}{\bar{\omega}_{1}} \arccos \left(\frac{-\mu \bar{\omega}_{1}^{4}+\left(\alpha^{2} \eta-H^{2} \mu+\bar{k}^{2} \beta^{2} \alpha I^{*} u^{*}\right) \bar{\omega}_{1}^{2}+\eta \alpha^{2} H^{2}-\bar{k}^{2} \beta^{2} \alpha^{2} H I^{*} u^{*}}{\eta\left[\bar{\omega}_{1}^{4}+\left(\alpha^{2}+H^{2}\right) \bar{\omega}_{1}^{2}+\alpha^{2} H^{2}\right]}+2 j \pi\right),
$$

where $j=0,1,2, \ldots$, then $\pm i \bar{\omega}_{1}$ is a pair of purely imaginary roots of (3.22) with $\bar{\tau}_{1}^{j}$.

Thus, we can define

$$
\bar{\tau}_{0}^{*}=\min \left\{\bar{\tau}_{1}^{j}\right\}
$$

Lemma 7. [9] Let $\lambda(\tau)=\alpha(\tau) \pm i \omega(\tau)$ be the root of Eq.(3.22) near $\tau=\bar{\tau}_{0}^{*}$ satisfying $\alpha\left(\bar{\tau}_{0}^{*}\right)=0, \omega\left(\bar{\tau}_{0}^{*}\right)=\bar{\omega}_{1}$. Suppose that $h^{\prime}\left(\bar{\omega}_{1}^{2}\right)>0$, where $h(z)$ is defined by (3.26). Then $\pm i \bar{\omega}_{1}$ 
is a pair of simple purely imaginary roots of Eq.(3.22). Moreover, the following transversality condition holds:

$$
\left.\frac{d(\operatorname{Re} \lambda(\tau))}{d \tau}\right|_{\tau=\bar{\tau}_{0}^{*}, \lambda=i \bar{\omega}_{1}}>0
$$

Applying Lemmas 5 7, it is easy to obtain the following conclusion.

Theorem 3. Let $\bar{\tau}_{0}^{*}$ be defined (3.28). Suppose that $\left(H_{1}\right)$ and $\left(H_{21}\right)$ hold.

(i) If the conditions of Lemma 6 are all satisfied, then the positive equilibrium point $E^{*}$ of system (3.1) is locally asymptotically stable when $\tau \in\left[0, \bar{\tau}_{0}^{*}\right)$.

(ii) If the conditions of (i) are satisfied, and $h^{\prime}\left(\bar{\omega}_{1}^{2}\right) \neq 0$, then system (3.1) undergoes a Hopf bifurcation at $E^{*}$ when $\tau=\bar{\tau}_{0}^{*}$. That is, system (3.1) has a branch of periodic solutions bifurcating from the positive equilibrium point $E^{*}$ near $\tau=\bar{\tau}_{0}^{*}$.

\section{Numerical Simulation and Discussion}

In this section, we simulate and analyze the spatial-temporal dynamic characteristics of the proposed model through simulations with Matlab, including the trend in the quantity and spatial distribution of the users on networks.

\subsection{Impact of the delay $\tau$ on the density of the users on networks}

Example 4.1.1. Consider system (2.1) with $r=0.5, T=5, \beta=0.1, \eta=0.1, \mu=0.35, d=$ $0.6, \bar{k}=5, K=0.4$. By a simple calculation, it is easy to obtain that the boundary equilibrium point is $E^{1}=(0.4,0)^{T}$. Obviously, the conditions $\left(H_{12}\right) \sim\left(H_{14}\right)$ hold. According to Theorem $1, E^{1}$ is locally asymptotically stable for $\forall \tau \geq 0$. Without loss of generality, we take $\tau=$ $5,15,25,35$. The simulation results are shown in Figure 1. From Figure 1 we notice that with increasing $\tau$, system (2.1) takes more time to converge to the boundary equilibrium point $E^{1}$. That is to say, the response time of the government feedback mechanism for rumour propagation directly influences the asymptotic convergence rate of system (2.1).

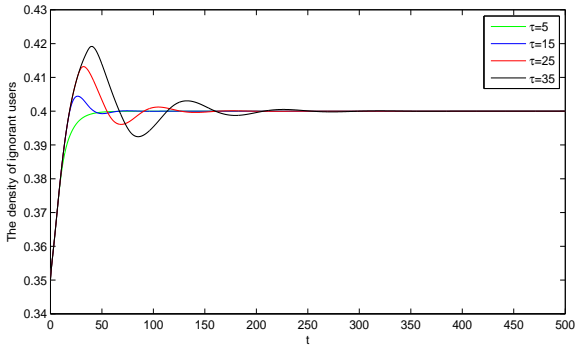

(a)

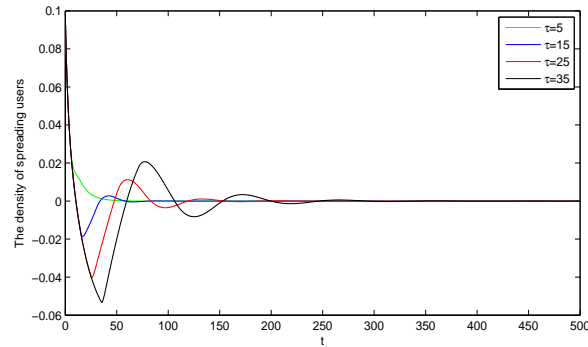

(b)

Figure 1: Impact of the time delay $\tau$ on the boundary equilibrium point $E^{1}$. (a) The density of ignorant users is locally asymptotically stable. (b) The density of spreading users is locally asymptotically stable. 
Example 4.1.2. Consider system (2.1) with the following parameters $r=0.5, T=\frac{5}{3}, \beta=$ $0.15, \eta=0.6, \mu=0.1, d=1.2, \bar{k}=7, K=0.4$. By calculating, the boundary equilibrium point is $E^{1}=(0.4,0)^{T}$ and the critical value is $\tau_{0}^{*}=3.2656$. Obviously, the parameters satisfy the conditions of Theorem 2. According to Theorem 2, system (2.1) is locally asymptotically stable at the boundary equilibrium point $E^{1}$ for $\tau=1.8,2.6,3 \in\left[0, \tau_{0}^{*}\right)$ and unstable for $\tau=3.4>\tau_{0}^{*}$, as Figure 2 shown. Furthermore, from Figure 2(a) and Figure 2(b) we notice that with increasing $\tau$, system (2.1) takes more time to converge to the boundary equilibrium point $E^{1}$. Then, when $\tau=3.4>\tau_{0}^{*}$, the spatially homogeneous periodic oscillations emerge from the boundary equilibrium point $E^{1}$ as shown in Figure 2(c) and Figure 2(d), which implies rumor spreads on a large scale in a short time and may destroy, block regular communications in online social networks, or even damage the real social stability. Therefore, it is clear that the response time of the government feedback mechanism for rumor propagation directly influences the asymptotic convergence rate of system (2.1), and also may lead to Hopf bifurcation (the phenomenon of the spatially homogeneous periodic oscillations).

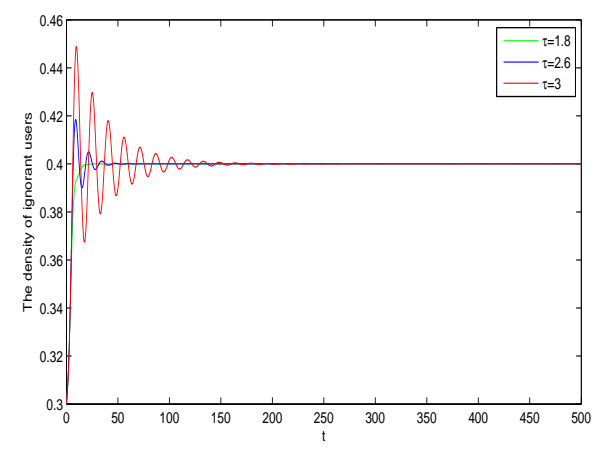

(a)

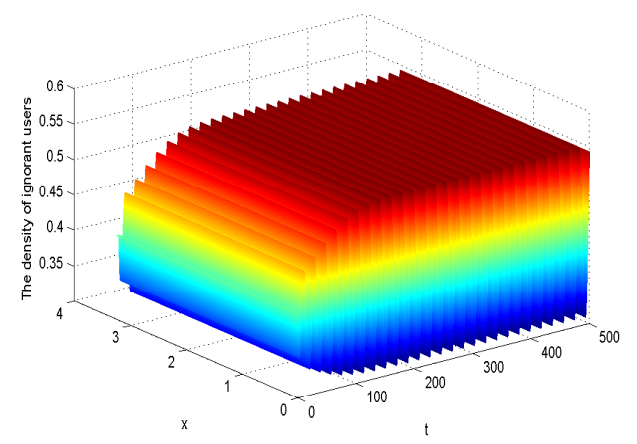

(c)

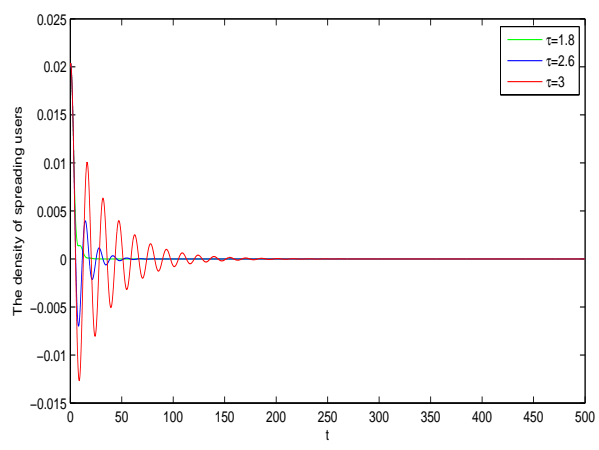

(b)

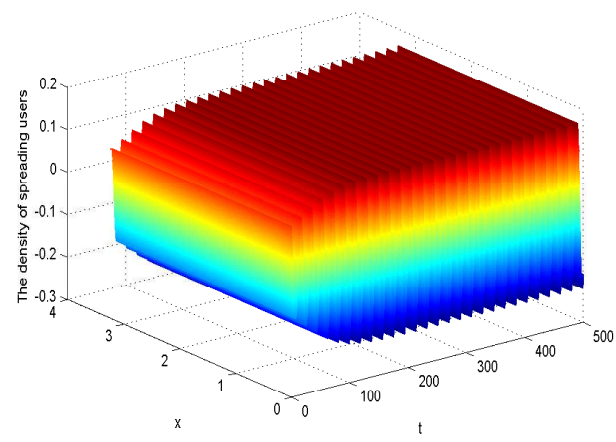

(d)

Figure 2: Impact of the time delay $\tau$ on the boundary equilibrium point $E^{1}$. (a) The density of ignorant users is locally asymptotically stable for $\tau=1.8,2.6,3<\tau_{0}^{*}$. (b) The density of spreading users is locally asymptotically stable for $\tau=1.8,2.6,3<\tau_{0}^{*}$. (c) Hopf bifurcation occurs from the density of ignorant users for $\tau=3.4>\tau_{0}^{*}$. (d) Hopf bifurcation occurs from the density of spreading users for $\tau=3.4>\tau_{0}^{*}$.

Example 4.1.3. Consider system (2.1) with the following parameters $r=0.3, T=2, \beta=$ $0.2, \eta=0.15, \mu=0.2, d=1.8, \bar{k}=8, K=0.4$. A simple calculation shows that the posi- 
tive equilibrium point is $E^{*}=(0.2187,0.0850)^{T}$ and the critical value is $\bar{\tau}_{0}^{*}=6.5653$. According to Theorem 3 , the positive equilibrium point $E^{*}$ is locally asymptotically stable for $\tau=3.5,4.5,5.5 \in\left[0, \bar{\tau}_{0}^{*}\right)$, as shown in Figure 3(a) and Figure 3(b). Taking $\tau=6.58>\bar{\tau}_{0}^{*}$, Figure 3(c) and Figure 3(d) show that the spatially homogeneous periodic solutions emerge from the positive equilibrium point $E^{*}$, which implies rumor explosively spreads in a short period and may destroy network stability and block regular communications in online social networks, or even cause a panic in the real society. Furthermore, from Figure 3(a) and Figure 3(b) we notice that with increasing $\tau$, system (2.1) takes more time to converge to the boundary equilibrium point $E^{*}$. Thus, the response time of the government feedback mechanism is sensitive to the stability of system (2.1).

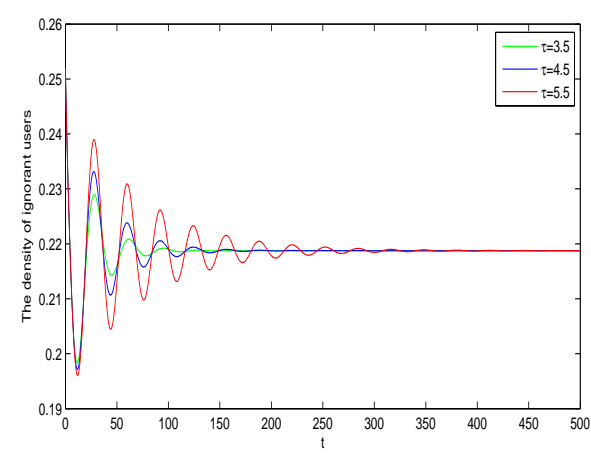

(a)

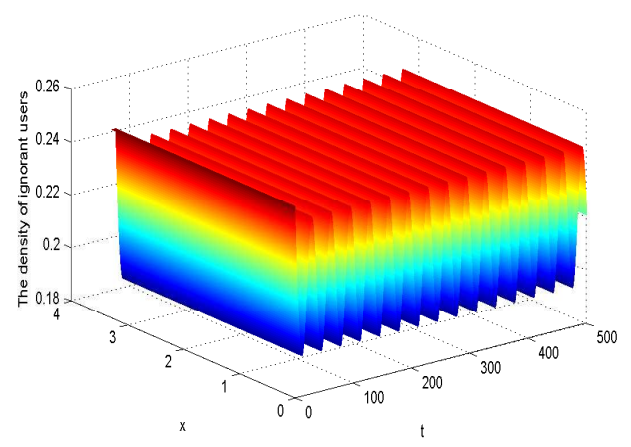

(c)

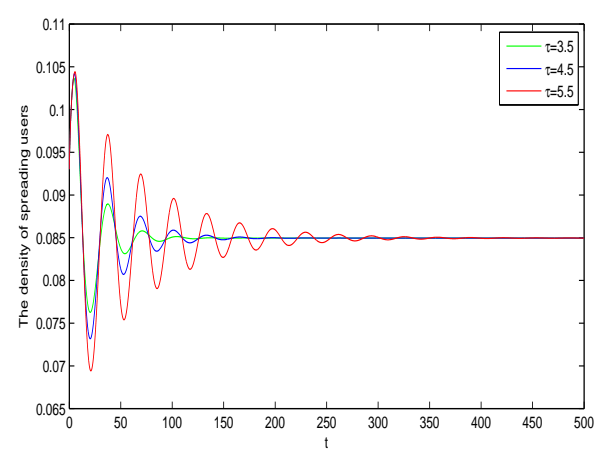

(b)

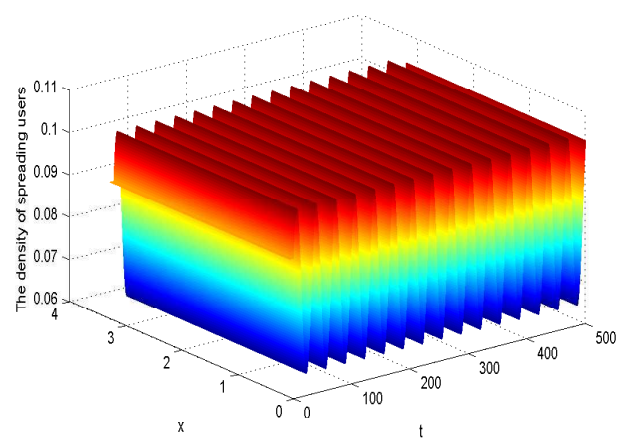

(d)

Figure 3: Impact of the time delay $\tau$ on the positive equilibrium point $E^{*}$. (a) The density of ignorant users is locally asymptotically stable for $\tau=3.5,4.5,5.5<\bar{\tau}_{0}^{*}$. (b) The density of spreading users is locally asymptotically stable for $\tau=3.5,4.5,5.5<\bar{\tau}_{0}^{*}$. (c) Hopf bifurcation occurs from the density of ignorant users for $\tau=6.58>\bar{\tau}_{0}^{*}$. (d) Hopf bifurcation occurs from the density of spreading users for $\tau=6.58>\bar{\tau}_{0}^{*}$.

Remark 4.1.1. According to Theorem 3, the delay $\tau$ can cause periodic oscillation at the positive equilibrium point $E^{*}$. That is to say, when $\tau>\bar{\tau}_{0}^{*}$, the densities of the users in online social networks begin to fluctuate with $\tau$ increases. Taking the parameters as the same as Example 3 and let $\tau \in[0,6.9]$, Figure 4 shows the density variations of the ignorant users and the spreading users, where the blue line reflects the minimum density and the red line reflects the maximum density. Figure 4 shows that when $\tau<6.5$ the maximum density and the minimum density of 
the two kinds of users are the same, which implies that, as time increases, the density will not change again. This phenomenon can be explained well from the theoretical analysis above. For example, we have obtained that when $\tau<6.5653$ the positive equilibrium point $E^{*}$ is locally asymptotically stable, that is, the density of the users remains unchanged at the end. On the other hand, as $\tau$ further enlarges, the maximum density increases and the minimum density decreases as shown in Figure 4. The reason of this phenomenon comes from the instability of $E^{*}$.

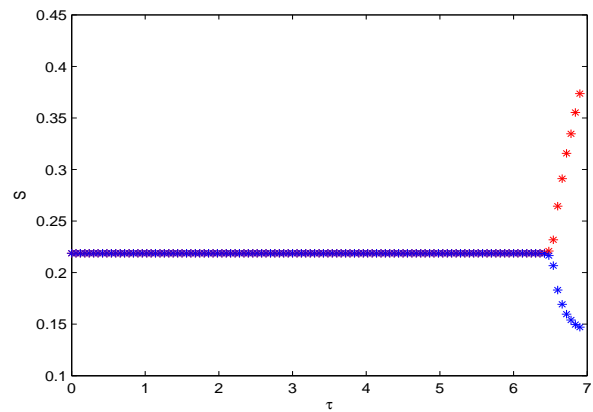

(a)

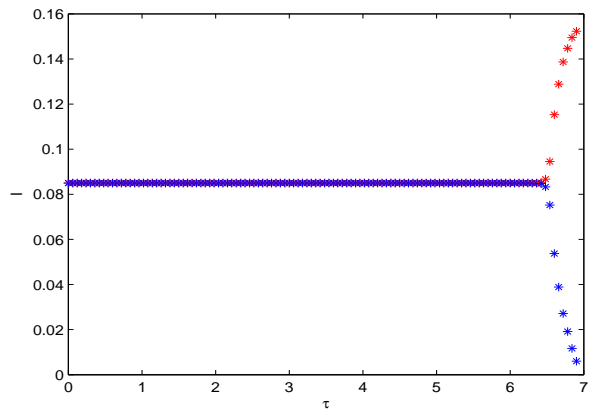

(b)

Figure 4: Minimum and maximum densities of the ignorant users and the spreading users for different time delays.

\subsection{Impact of the network structure $\bar{k}$ on the density of the users on networks}

Example 4.2.1. To observe the impact of different network structures on the density of the users, we choose $r=0.5, T=5, \beta=0.1, \eta=0.1, \mu=0.6, d=0.6, K=0.4$, and assign 1, 6 and 12 to $\bar{k}$, respectively. By calculating, we can easily obtain that the boundary equilibrium point $E^{1}=(0.4,0)^{T}$ is locally asymptotically stable for $\forall \tau \geq 0$. Let $\tau=1$, Figure 5 shows that with $\vec{k}$ increasing, system (2.1) takes more time to converge to the boundary equilibrium point $E^{1}$. That is to say, if a spreading use owns more neighbor users, then the rumor will gradually increase its impact on the asymptotic convergence rate of system (2.1).

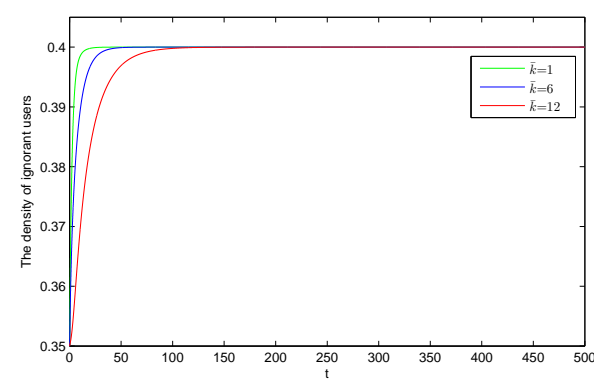

(a)

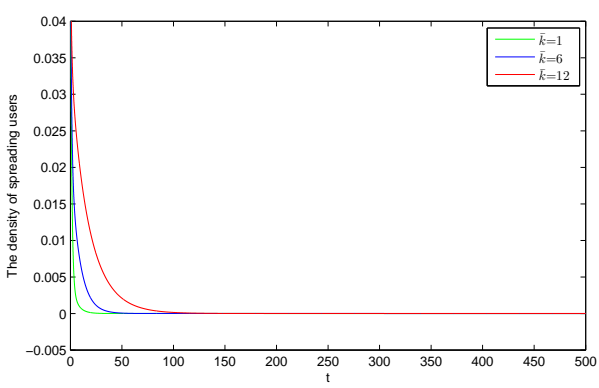

(b)

Figure 5: Impact of network structure on the boundary equilibrium point $E^{1}$. (a) The density of ignorant users is locally asymptotically stable with increasing $\vec{k}$ for $\tau=1$. (b) The density of spreading users is locally asymptotically stable with increasing $\bar{k}$ for $\tau=1$. 


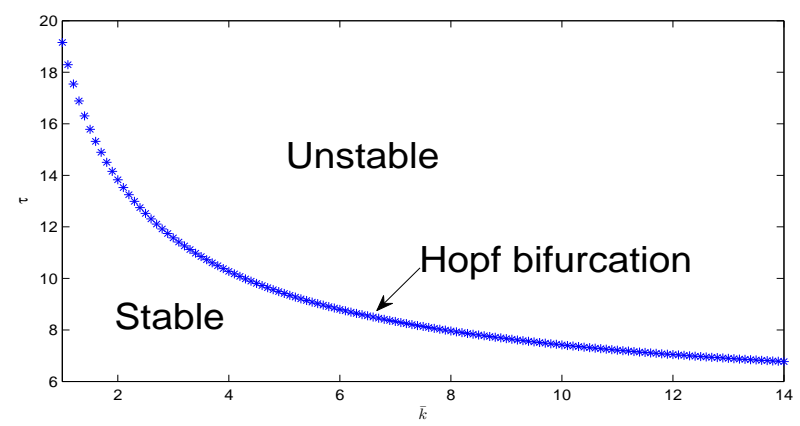

Figure 6: The region of stability of the boundary equilibrium point $E^{1}$ varies with network structure $\bar{k}$ increasing.

Example 4.2.2. Take $r=0.3, T=\frac{5}{3}, \beta=0.1, \eta=0.3, \mu=0.3, d=1, K=0.4$, and vary $\bar{k}$ from 1 to 14 continuously in system (2.1). In this part, we discuss the effect of network structure $\bar{k}$ on the region of stability for the boundary equilibrium point $E^{1}=(0.4,0)^{T}$, as shown in Figure 6. From Figure 6, we notice that with increasing $\bar{k}$ the region of stability for system (2.1) is decreasing. This means that with increasing interrelationship between users, rumor is more easy to propagate on networks. In this situation, rumor propagation is a serious threat to network security.

Example 4.2.3. Keep the parameters $r=0.3, T=\frac{5}{3}, \beta=0.3, \eta=0.1, \mu=0.1, d=1.8, K=$ 0.8 , and $\bar{k}$ varies in $[1,12]$. According to $\left(H_{1}\right)$, the corresponding positive equilibrium points $E^{*}$ are shown in Figure 7. Figure 7(a) shows that with the increase of network structure $\vec{k}$, the density of ignorant users is decreasing. From Figure 7(b) we notice that the density of spreading users initially is increasing and then it is decreasing gradually. This is, we believe, initially a spreading user with a complex relationship on networks may communicate with more ignorant users, which leads to more ignorant users being infected and more spreading users occurring. Then, due to the government feedback mechanism, the density of spreading users will gradually decrease.

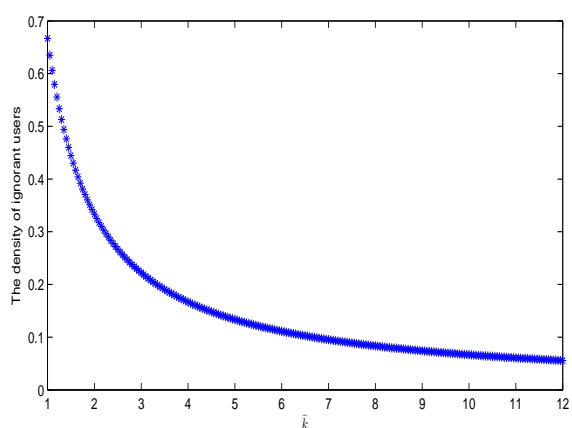

(a)

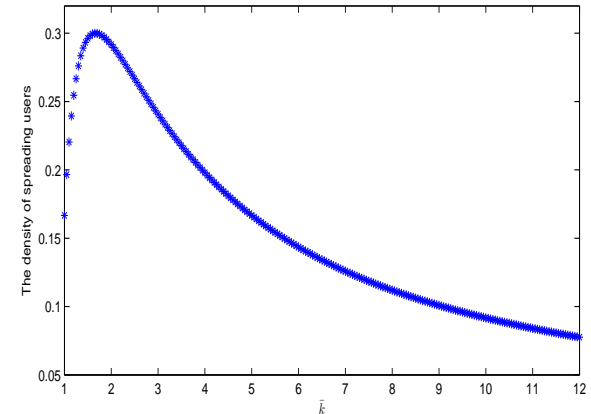

(b)

Figure 7: The positive equilibrium point $E^{*}$ of system (2.1) varies with increasing $\bar{k}$. 


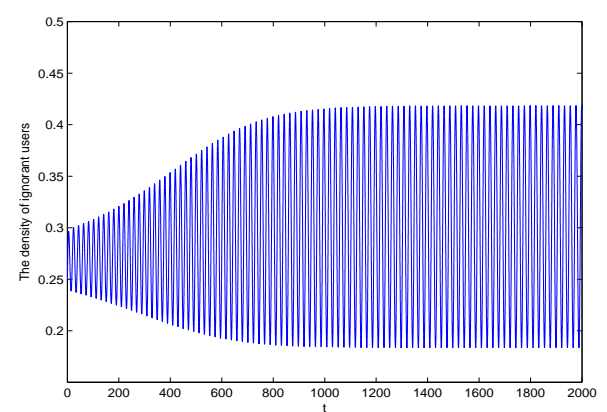

(a)

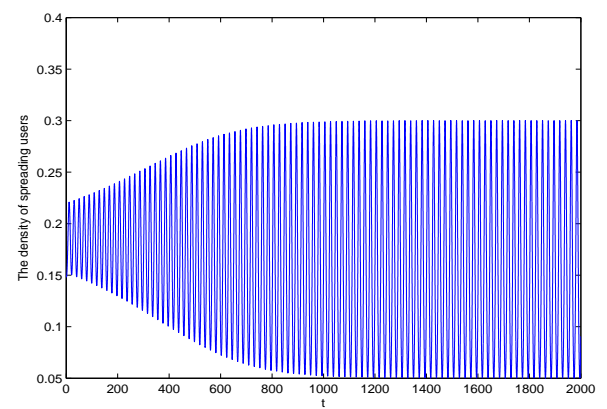

(b)

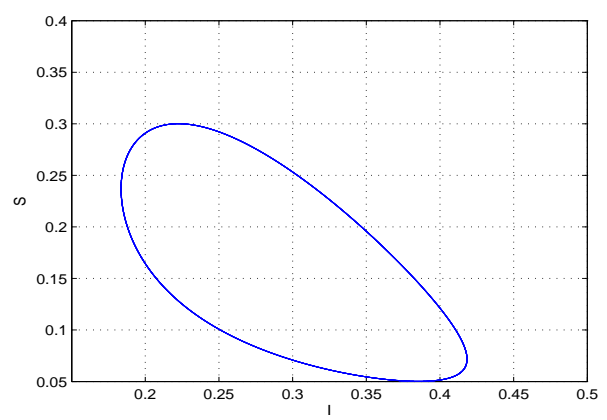

(c)

Figure 8: Hopf bifurcation occurs from the positive equilibrium point $E^{*}$ for $T=0.5$.

\subsection{Impact of the spatial-temporal delay $T$ on the density of the users on networks}

Example 4.3.1. To observe the impact of the spatial-temporal delay $T$ on system (2.1), we consider $r=0.6, \beta=0.3, \eta=0.2, \mu=0.2, \bar{k}=5, K=0.5, d=1.8, \tau=4.1$, and assign 0.5 and 2.5 to $T$, respectively. A simple calculation shows that the positive equilibrium point is $E^{*}=(0.2667,0.1867)^{T}$. Obviously, the spatial-temporal delay $T$ does not change the positive equilibrium point $E^{*}$. Furthermore, according to Theorem 3, it follows that the positive equilibrium point $E^{*}$ is unstable as $T=0.5$ (See Figure 8) and it is stable as $T=2.5$ (See Figure 9). That is, the spatial-temporal delay $T$ can change the stability of the positive equilibrium point $E^{*}$. 


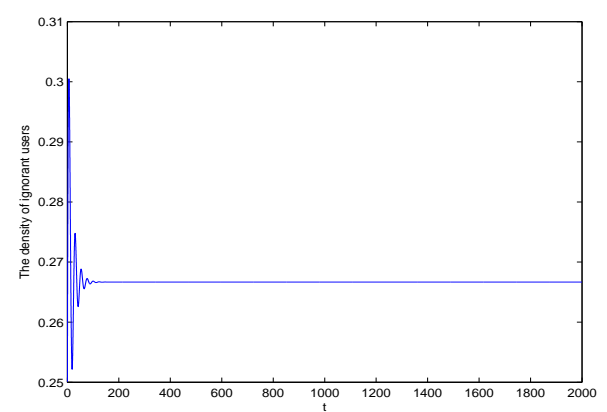

(a)

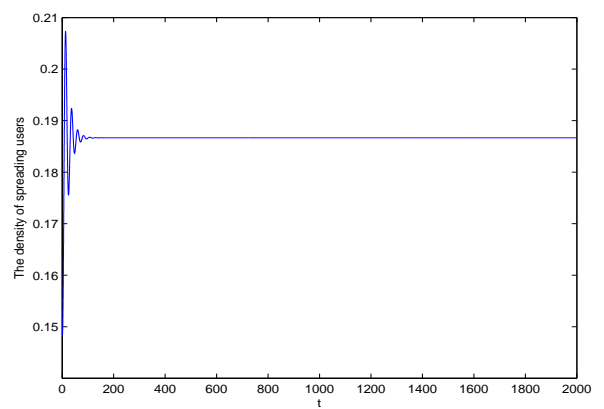

(b)

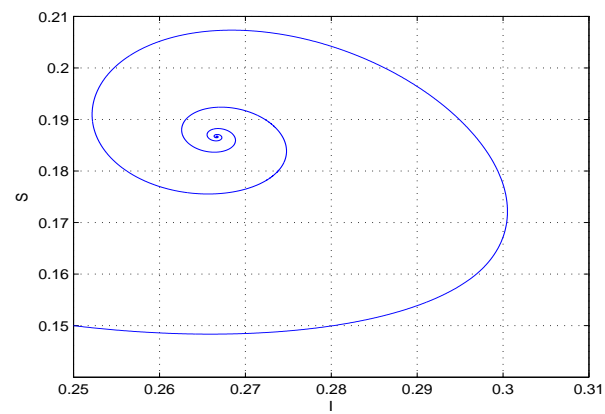

(c)

Figure 9: The positive equilibrium point $E^{*}$ is locally asymptotically stable for $T=2.5$.

Remark 4.3.1. Take the parameters $r=0.6, \beta=0.3, \eta=0.2, \mu=0.2, \bar{k}=5, K=0.5, d=$ $1.8, \tau=3$, and choose $T=0.5$ and 2.5, respectively. Figure (10) shows that when $T=0.5$ the convergence time of system (2.1) is longer than that of the situation $T=2.5$. This means that the spatial-temporal delay $T$ can also effectively change the convergence time of system (2.1). 


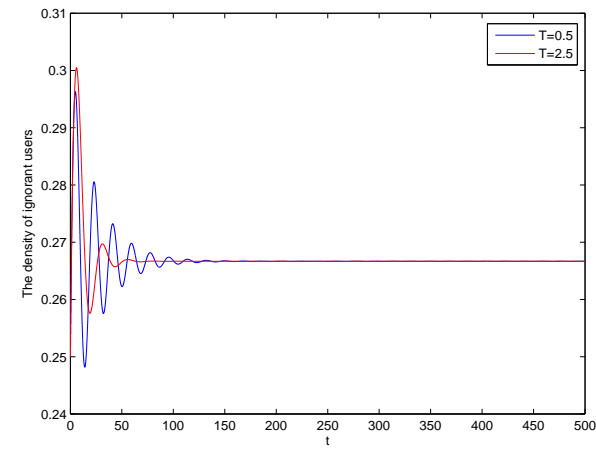

(a)

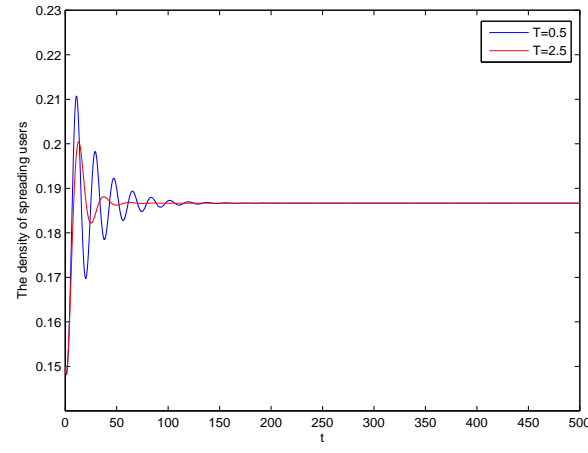

(b)

Figure 10: The positive equilibrium point $E^{*}$ of system (2.1) is locally asymptotically stable with $T=0.5$ and $T=2.5$.

\subsection{Sensitivity analysis for the density of spreading users on networks}

In real world applications, our main objective is to control the density of spreading users in a low state. Thus, we consider the influence of the parameters of system (2.1) on rumor diffusion; in other words, we study the relationship between the density of spreading users and the parameters in our model. By some calculations, it is obvious that

$$
\frac{\partial S}{\partial \mu}=-\frac{r}{\bar{k}^{2} \beta^{2} K}<0, \quad \frac{\partial S}{\partial \eta}=-\frac{r}{\bar{k}^{2} \beta^{2} K}<0,
$$

while

$$
\frac{\partial S}{\partial r}=\frac{\bar{k} \beta K-\mu-\eta}{\bar{k}^{2} \beta^{2} K}>0, \quad \frac{\partial S}{\partial K}=\frac{r(\mu+\eta)}{\bar{k}^{2} \beta^{2} K^{2}}>0 .
$$

It can be seen that, among these parameters, $S(t, x)$ is a decreasing function of $\mu$ and $\eta$. As opposed to this, $r$ and $K$ have a directly proportional relationship with $S$. Figure 11 shows that the density of spreading users varies with increasing the parameters $r, K, \mu, \eta$. Therefore, in order to control the density of spreading users in a low state, a government should take some effective measures to strengthen the network management and build a good network environment (It is beneficial to decrease the parameters $K$.). On the other hand, by increasing the parameters $\mu$ and $\eta$ or decreasing $r$, we can also control rumor diffusion on networks. A government may need to take effective measures to popularize the knowledge of network information security and control rumor propagation actively. 


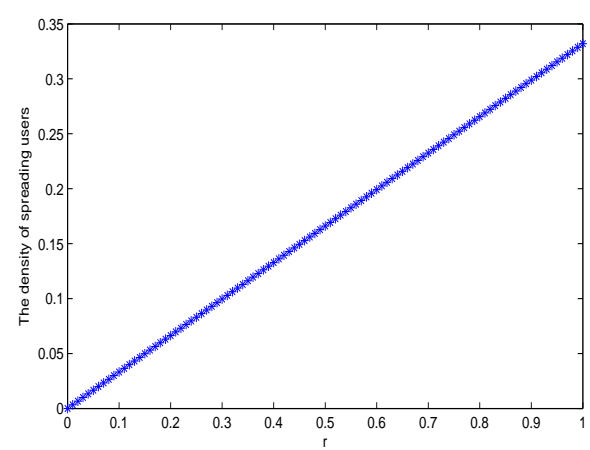

(a)

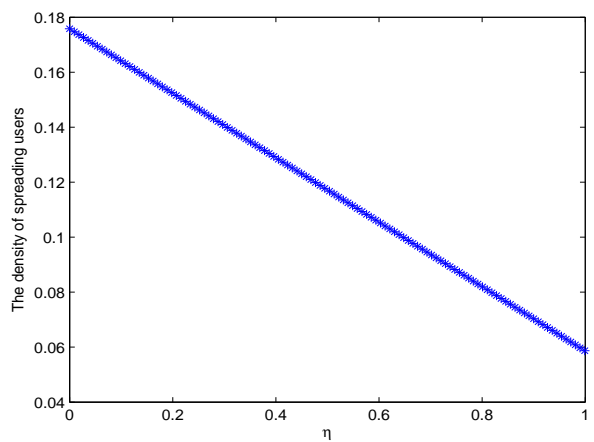

(c)

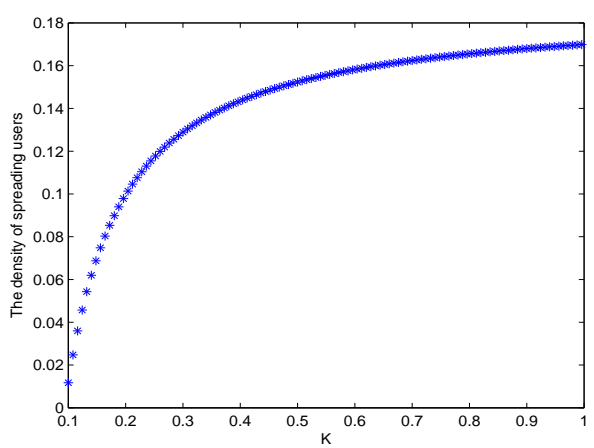

(b)

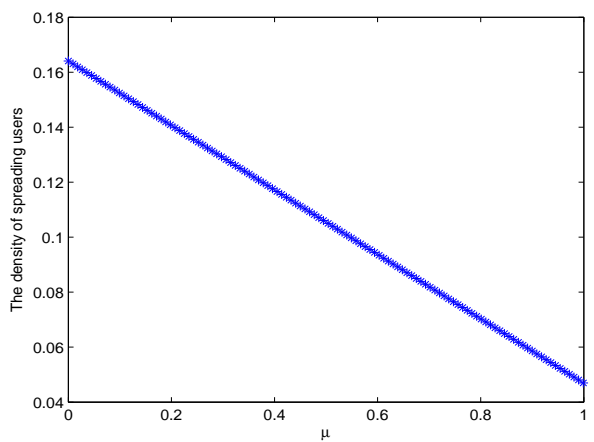

(d)

Figure 11: Sensitivity analysis for the density of spreading users on networks. (a) The density of spreading users varies with increasing $r$. (b) The density of spreading users varies with increasing $K$. (c) The density of spreading users varies with increasing $\eta$. (d) The density of spreading users varies with increasing $\mu$.

Remark 4.4.1. In the above numerical simulations, through artificial selection of parameters, we have verified the accuracy of the theories and analyzed the inherent characteristics of rumor propagation. However, the experiments by using real data are still very difficult and challenging for our proposed model with the discrete and nonlocal delays at present. For example,

(1) There are no data to measure the spatial-temporal delay $T$ at present. Because different infected users to develop the real spreading users may take different time, it is difficult to give an exact value for $T$.

(2) In our model, there exists a term $-\mu S(t, x)$, which means the spreading users leaves the networks because of the impact of the process of forgetting. It is well known that the forgetting process is subjective behavior, and it is difficult to describe the process of forgetting quantitatively by existing data.

With the further development of big data, we will improve our work on the experiments with real data in a future work. 


\section{Conclusion and Future Work}

Based on reaction-diffusion equations, in this paper we propose a spatial-temporal dynamic model with discrete and nonlocal delays for investigating the spatial-temporal dynamics of rumor propagation in online social networks. Applying the theorem of PFDEs, we present verifiable conditions for stability and Hopf bifurcation of the boundary equilibrium point $E^{1}$ and the positive equilibrium point $E^{*}$ for system (2.1). Furthermore, by sensitivity analysis for the density of spreading users, we obtain the parameters $\mu, \eta, r$ and $K$ are sensitive to rumor propagation. Numerical simulations reveal that the discrete delay is responsible for the stability switch of system (2.1), and a Hopf bifurcation occurs when the discrete delay passes through a certain threshold. Moreover, by numerical simulations, we discuss the impact of the spatial-temporal delay $T$ on system (2.1). Thus, reaction-diffusion modeling, which predicts both the temporal dynamic behavior and the spatial distribution of rumor propagation, provides a new insight into rumor propagation in online social networks.

Owing to the characteristics of the large-scale applications for online social networks, sometimes it is difficult to grasp the initial density of network users. Thus, further discussion of the global stability for this model is significant. At the same time, with the rapid development of online social networks, all kinds of rumors threaten network security. Considering the synchronization between all kinds of rumor propagations, this presents a variety of pragmatic interests. In addition, more and more researchers, based on the ODE epidemic-like model, have begun to focus on rumor propagation on an inhomogeneous social network [24, 42] (such as scale-free networks [14]), which maybe more complex and realistic. But, as far as we know, analyzing a PDE rumor propagation model on an inhomogeneous social network is still a challenge. Because of the length limitation for this paper, we will discuss these problems further in future.

\section{Acknowledgement}

The work is partially supported by National Natural Science Foundation of China under Grant 61174155 and 11571170, and the Qing Lan Project of Jiangsu. The work is also sponsored by Funding of Jiangsu Innovation Program for Graduate Education KYZZ15_0091, the Fundamental Research Funds for the Central Universities. Additionally, the authors would like to express their gratitude to the editor and the anonymous reviewers for their valuable comments and suggestions.

\section{References}

[1] F. Buccafurri, G. Lax, S. Nicolazzo, A. Nocera, A model to support design and development of multiple-socialnetwork applications, Inform. Sci. 331 (2016) 99-119.

[2] D.J. Daley, D.G. Kendall, Epidemics and rumours, Nature 204 (4963) (1964) 1118

[3] D.J. Daley, D.G. Kendall, Stochastic rumours, IMA J. Appl. Math. 1 (1) (1965) 42-55.

[4] F. Fu, X. Chen, L. Liu, L. Wang, Social dilemmas in an online social network: the structure and evolution of cooperation, Phys. Lett. A 371 (1-2) (2007) 58-64.

[5] S.A. Gourley, J. W. H. So, Dynamics of a food-limited population model incorporating nonlocal delays on a finite domain, J. Math. Biol. 44 (1) (2002) 49-78.

[6] A. Grabowski, N. Kruszwska, R.A. Kosinki, Dynamic phenomena and human activity in an artificial society, Phys. Rev. E 78 (2008) 066110. 
[7] F. Ha, S.W. Kim, C. Faloutsos, S. Park, An analysis on information diffusion through BlogCast in a blogosphere, Inform. Sci. 290 (2015) 45-62.

[8] H. Hayakawa, Ryugen no shakaigaku-keishikisyakaigaku karano sekkin (sociology of rumor-approach from formal sociology). Seikyusya, Tokyo, Japan, 2002.

[9] H.J. Hu, L.H. Huang, Stability and Hopf bifurcation analysis on a ring of four neurons with delays, Appl. Math. Comput. 213 (2) (2009) 587-99.

[10] L.A. Huo, P.Q. Huang, X. Fang, An interplay model for authoritiesactions and rumor spreading in emergency event, Physica A 390 (20) (2011) 3267-3274.

[11] J.L. Iribarren, E. More, Impact of human activity patterns on the dynamics of information diffusion, Phys. Rev. Lett. 103 (3) (2009) 038702.

[12] M. Jankowski-Lorek, S. Jaroszewicz, L. Ostrowski, A. Wierzbicki, Verifying social network models of Wikipedia knowledge community, Inform. Sci. 339 (2016) 158-174.

[13] Y. Li, M.J. Qian, D.P. Jin, P. Hui, A.V. Vasilakos, Revealing the efficiency of information diffusion in online social networks of microblog, Inform. Sci. 293 (2015) 383-389.

[14] W.H. Li, S.T. Tang, S. Pei, S. Yan, S.J. Jiang, X. Teng, Z.M. Zheng, The rumor diffusion process with emerging independent spreaders in complex networks, Physica A, 397 (3) (2014) 121-128.

[15] T. Li, Y.M. Wang, Z.H. Guan, Spreading dynamics of a SIQRS epidemic model on scale-free networks, Commun. Nonlinear Sci. Numer. Simul. 19 (3) (2014) 686-692.

[16] C.C. Lin, J.R. Kang, J.Y. Chen, An integer programming approach and visual analysis for detecting hierarchical community structures in social networks, Inform. Sci. 299 (2015) 296-311.

[17] P.G. Lind, L.R. da Silva, J.J.S. Andrade, H.J. Herrmann, Spreading gossip in social networks, Phys. Rev. E 76 (2007) 036117

[18] C. Liu, Z.K. Zhang, Information spreading on dynamic social networks, Commun. Nonlinear Sci. Number. Simul. 19 (3) (2014) 896-904.

[19] J. Ma, D.D. Li, Z.H. Tian, Rumor spreading in online social networks by considering the bipolar social reinforcement, Physica A 447 (2015) 108-115.

[20] D.P. Maki, M. Thomson, Mathematical Models and Applications, with Emphasis on Social, Life, and Management Sciences, Prentice-Hall, Englewood Cliffs, NJ, 1973

[21] Y. Moreno, M. Nekovee, A. Pacheco, Dynamics of rumor spreading in complex networks, Phys. Rev. E 64 (2004) 066130

[22] Y. Moreno, M. Nekovee, A. Vespignani, Efficiency and reliability of epidemic data dissemination in complex networks, Phys. Rev. E 64 (2004) 055101.

[23] M. Nekovee, Y. Moreno, G. Bianconi, M. Marisili, Theory of rumour spreading in complex social networks, Physica A, 374 (1) (2007) 457-470.

[24] F. Roshani, Y. Naimi, Effects of degree-biased transmission rate and nonlinear infectivity on rumor spreading in complex social networks, Phys. Rev. E, 85 (2012) 036109.

[25] K. Saito, M. Kimura, K. Ohara, H. Motoda, Super mediator-A new centrality measure of node importance for information diffusion over social network, Inform. Sci. 329 (2016) 985-1000.

[26] X.Y. Shi, J.A. Cui, X.Y. Zhou, Stability and hopf bifurcation analysis of an eco-epidemic model with a stage structure, Nonlinear Anal. Theory, Methods Appl. 74 (4) (2011) 1088-1106.

[27] T. Shibutani, Improvised News: A Sociological Study of Rumor, Bobbs-Merrill, Indianapolis, America, 1966.

[28] S.A. Thomas, Lies, damn lies, and rumors: an analysis of collective efficacy, rumors, and fear in the wake of Katrina, Sociol. Spectrum 27 (6) (2007) 679-703

[29] C.R. Tian, L Zhang, Z Ling, The stability of a diffusion model of plankton allelopathy with spatio-temporal delays, Nonlinear Anal. Real World Appl. 10 (4) (2009) 2036-2046.

[30] C. Wang, C.Y. Liu, Y.P Hu, Z.H. Liu, J.F. Ma, Stability of information spreading over social network, Acta Phys. Sin. 63 (18) (2014) 180501.

[31] F. Wang, H.Y. Wang, K. Xu, Diffusive logistic model towards predicting information diffusion in online social networks, in: Proceedings of 32nd International Conference on Distributed Computing Systems Workshops (ICDCSW 2012) IEEE Press, 2012, pp. 133-139.

[32] F. Wang, H.Y. Wang, K. Xu, J.H. Wu, X.H. Jia, Characterizing Information Diffusion in Online Social Networks with Linear Diffusive Model, in: Proceedings of 33rd International Conference on Distributed Computing Systems Workshops (ICDCSW 2013) IEEE Press, 2013, pp. 307-316.

[33] J.J. Wang, L.J. Zhao, R.B. Huang, SIRaRu rumor spreading model in complex networks, Physica A 398 (1) (2014) $43-55$.

[34] L.L. Xia, G.P. Jiang, B. Song, Y.R. Song, Rumor spreading model considering hesitating mechanism in complex social networks, Physica A 437 (2015) 295-303.

[35] F. Xiong, Y. Liu, Z.J. Zhng, J. Zhu, Y. Zhang, An information diffusion model based on retweeting mechanism for online social media, Phys. Lett. A 376 (30-31) (2012) 2103-2108. 
[36] R. Xu, M.A.J. Chaplain. F.A. Davidson, Global convergence of a reaction-diffusion predator-prey model with stage structure and nonlocal delays, Comput. Math. Appl. 53 (5) (2007) 770-778.

[37] R. Xu, Z.E. Ma, Global stability of a SIR epidemic model with incidence rate and time delay, Nonlinear Anal. Real World Appl. 10 (5) (2009) 3175-3189.

[38] Z. Yu, C. Wang, J.J. Bu, X. Wang, Y. Wu, C. Chen, Friend recommendation with content spread enhancement in social networks, Inform. Sci. 309 (2015) 102-118.

[39] D.H. Zanette, Dynamics of rumor propagation on small-world networks, Phys. Rev. E 65 (2002) 044908.

[40] D.H. Zanette, Critical behavior of propagation on small-world networks, Phys. Rev. E 64 (2001) 050901.

[41] T.L. Zhang, Z.D. Teng, Global behavior and permanence of SIRS epidemic model with time delay, Nonlinear Anal. Real World Appl. 9 (4) (2008) 1409-1424.

[42] Y.C. Zhang, S. Zhou, Z.Z. Zhang, J.H. Guan, S.G. Zhou, Rumor evolution in social networks, Phys. Rev. E 87 (2013) 030133

[43] L.J. Zhao, H.X. Cui, X.Y Qiu, X.L. Wang, J.J Wang, SIR rumor spreading model in the new media age, Physica A 392 (4) (2013) 995-1003.

[44] L.J. Zhao, J.J. Wang, Y.C. Chen, Q. Wang, J.J. Cheng, SIHR rumor spreading model in social networks, Physica A 391 (7) (2012) 2444-2453.

[45] L.J. Zhao, Q. Wang, J.J. Cheng, Y.C. Chen, J.J. Wang, W. Huang, Rumor spreading model with consideration of forgetting mechanism: a case of online blogging LiveJournal, Physica A 390 (13) (2011) 2619-2625.

[46] L.J. Zhao, Q. Wang, J.J. Cheng, D. Zhang, T. Ma, Y.C. Chen, J.J. Wang, The impact of authorities media and rumor dissemination on the evolution of emergency, Physica A 391 (15) (2012) 3978-3987.

[47] L.J. Zhao, W.L. Xie, H. Gao, X.Y. Qiu, X.L. Wang, S.H. Zhang, A rumor spreading model with variable forgetting rate, Physica A 392 (23) (2013) 6146-6154.

[48] X.Y. Zhou, J.A. Cui, Analysis of stability and bifurcation for an SEIR epidemic model with saturated recovery rate, Commun. Nonlinear Sci. Number. Simul. 16 (11) (2011) 4438-50.

[49] J. Zhou, Z.H. Liu, B.W. Li. Influence of network structure on rumor propagation, Phys. Lett. A 368 (6) (2007) $458-463$.

[50] L.H. Zhu, H.Y. Zhao, Dynamical analysis and optimal control for a malware propagation model in an information network, Neurocomputing 149 (C) (2015) 1370-1386.

[51] L.H. Zhu, H.Y. Zhao, H.Y. Wang, Bifurcation and control of a delayed diffusive logistic model in online social networks, in: Proceedings of 33rd Chinese Control Conference (CCC 2014) IEEE Press, 2014, pp. 2773-2778.

[52] L.H. Zhu, H.Y Zhao, X.M. Wang, Bifurcation analysis of a delay reaction-diffusion malware propagation model with feedback control, Nonlinear Sci. Number. Simul. 22 (1-3) (2015) 747-768. 